\title{
Analysis of EB Support Saddles and Forces Between Modules during Assembly
}

\author{
By \\ V. Guarino
}

Argonne National Laboratory

High Energy Physics Division

Argonne, IL 60439

July 16, 2002

*Work supported in part by the U.S. Department of Energy, Division of High Energy Physics, under Contract W-31-109-ENG-38. 
Argonne National Laboratory, with facilities in the states of Illinois and Idaho, is owned by the United States Government and operated by The University of Chicago under the provisions of a contract with the Department of Energy.

\section{DISCLAIMER}

This report was prepared as an account of work sponsored by an agency of the United States Government. Neither the United States Government nor any agency thereof, nor The University of Chicago, nor any of their employees or officers, makes any warranty, express or implied, or assumes any legal liability or responsibility for the accuracy, completeness, or usefulness of any information, apparatus, product, or process disclosed, or represents that its use would not infringe privately owned rights. Reference herein to any specific commercial product, process, or service by trade name, trademark, manufacturer, or otherwise, does not necessarily constitute or imply its endorsement, recommendation, or favoring by the United States Government or any agency thereof. The views and opinions of document authors expressed herein do not necessarily state or reflect those of the United States Government or any agency thereof, Argonne National Laboratory, or The University of Chicago.

Available electronically at http://www.doe.gov/bridge

Available for a processing fee to U.S. Department of Energy and its contractors, in paper, from:

U.S. Department of Energy

Office of Scientific and Technical Information

P.O. Box 62

Oak Ridge, TN 37831-0062

phone: (865) 576-8401

fax: (865) 576-5728

email: reports@adonis.osti.gov 
ANL-HEP-TR-02-058

\title{
Analysis of EB Support Saddles and Forces Between Modules during Assembly
}

\author{
By \\ V. Guarino
}

Argonne National Laboratory High Energy Physics Division

Argonne, IL 60439

July 16, 2002

*Work supported in part by the U.S. Department of Energy, Division of High Energy Physics, under Contract W-31-109-ENG-38. 


\section{Analysis of EB Support Saddles and Forces Between Modules during Assembly \\ Victor Guarino \\ Argonne National Laboratory \\ July 16, 2002}

\section{Introduction}

As the EB Tile Calorimeter is constructed, the support saddles and the modules will be subjected to different forces, stresses, and deflections than when completely assembled. The purpose of this analysis is to examine the forces, stresses, and deflections acting on the support saddles and modules at various stages of assembly.

\section{Model Description}

In the FE model of the saddle and back cryostat support, the $\mathrm{Z}$ axis ran along the beam line, the $\mathrm{Y}$ axis was in the vertical direction, and the $\mathrm{X}$ axis was horizontal.

Software used: COSMOS/M and Solid Works

Type of Elements: Solid

Number of Elements: 78,355

Material: Stainless Steel, E $=206,000 \mathrm{MPa}$

Code applied: Euro code 3

A complete description of how the modules, connections within and between modules, and support saddles were modeled can be found in ANL Technical Report ANL-HEP-TR-01-097, "Extended Barrel Support Saddle Design and Analysis".

\section{Load Cases}

There were eight load cases examined to date. All of the loads are at their nominal values. These are described below.

EB1 - 63 modules with cryostat load

EB2 - 63 modules without cryostat load

EB3 - 48 modules with cryostat load

EB4 - 48 modules without cryostat load

EB5 - 32 modules with cryostat load

EB6 - 32 modules without cryostat load

EB7 - 24 modules with cryostat load

EB8 - 24 modules without cryostat load 
EB9 - 14 modules with saddles

EB10 - 14 modules with saddles and support cradle

EB11 - 14 modules without saddles but with support cradle only

Load Case EB7 did not converge to a solution. It appears that this configuration is unstable without any further supports.

Figure 1 below shows an example of how modules are numbered.

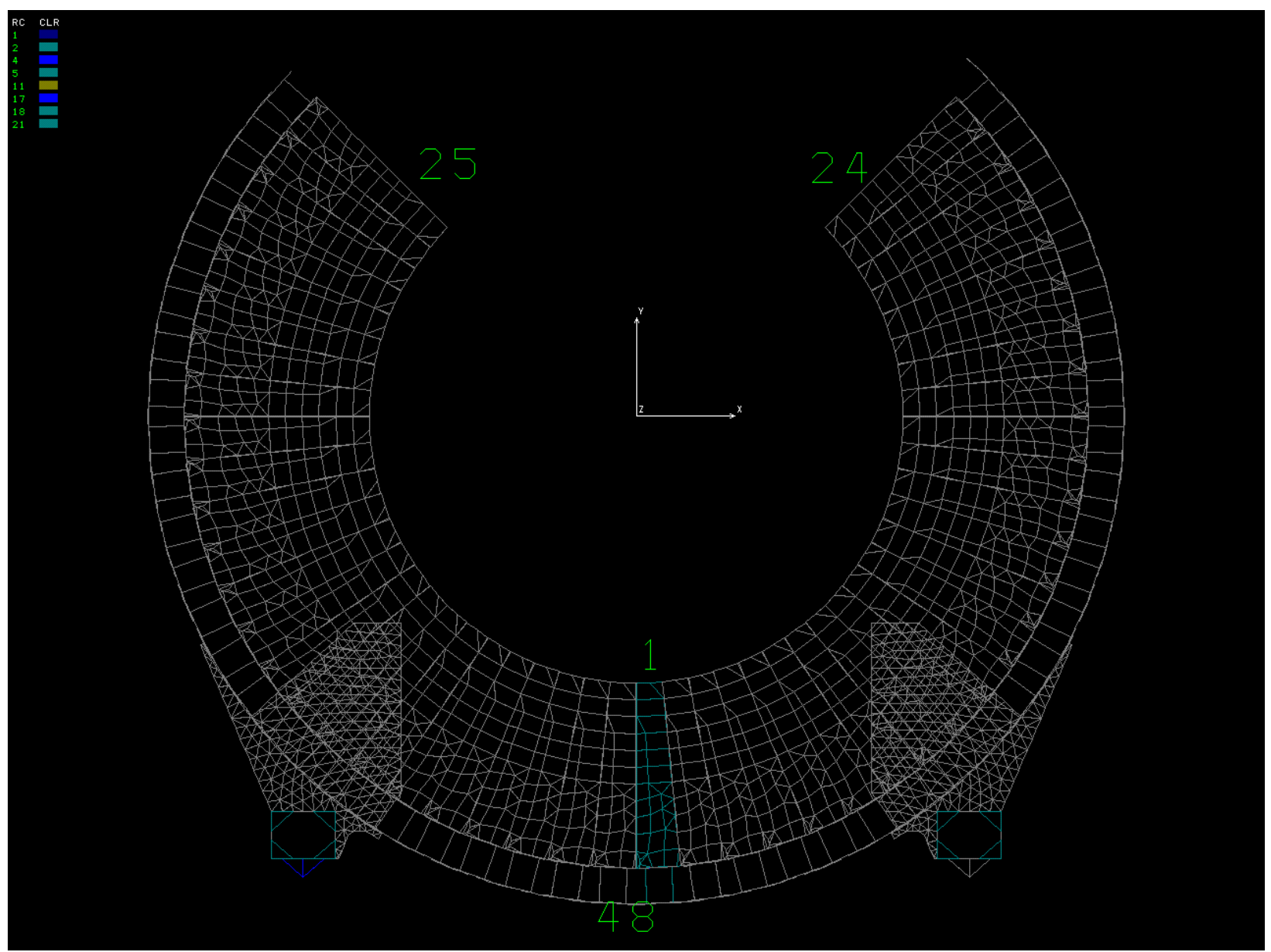

Fig. 1. Module numbering scheme.

\section{Stability of Assembly}

There are two load cases where the EB assembly is not stable and additional supports are needed; load case EB7, which has 24 modules in place and the cryostat load first applied and, load case 9 , in which there are 14 modules supported only by the saddle. The FE model in both of these load cases found a solution; however, the deflections were excessively large, because the entire structure had to deform in order to find a static solution to the problem. Also, in both of these 
load cases, when a small force (100N) was applied to the back cryostat support in the $\mathrm{X}$ direction, the deflections and forces that were found by the FE model changed dramatically.

Both of these load cases were examined by performing some simple hand calculations and with a 2D FORTRAN program, which solved for the reaction forces on the saddle. With the constraint that the swivel bolt forces must be in compression in both of these load cases, it was found that a static solution was only possible if additional support was provided to the saddles or modules below the saddle. Each of the unstable load cases will be examined in detail below.

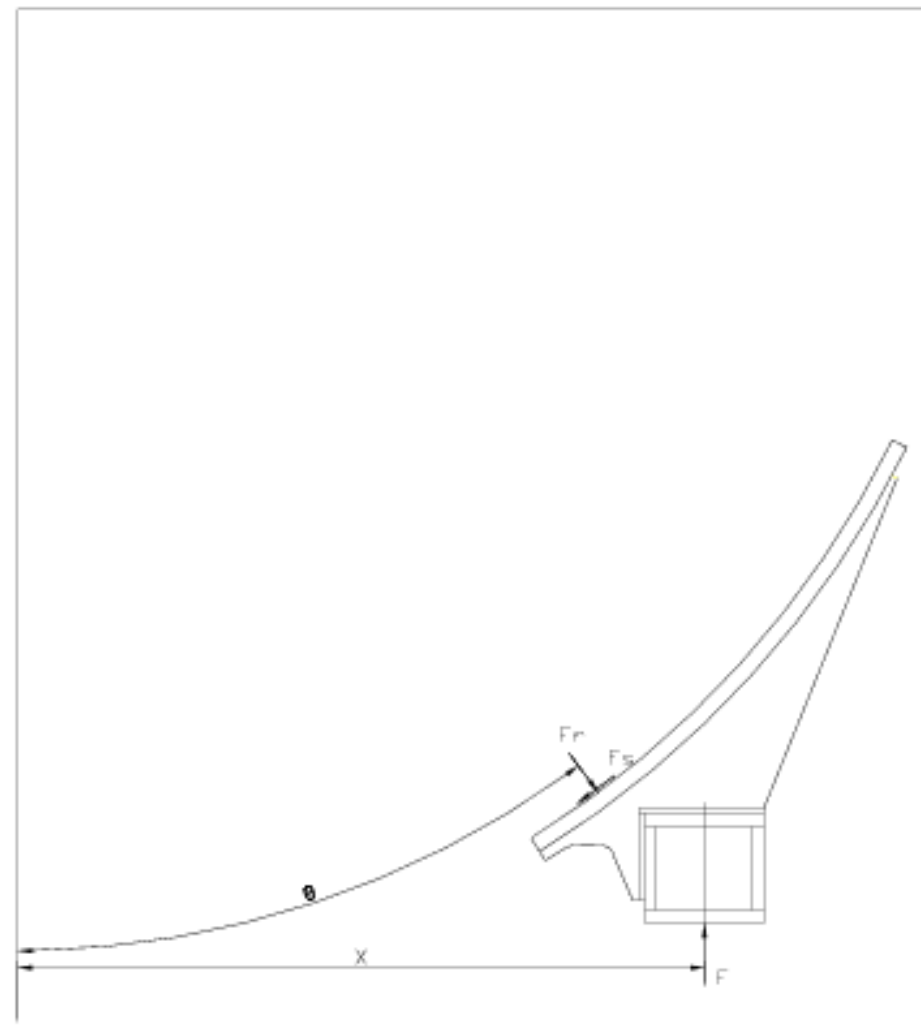

Fig. 2. Static Analysis of Load Case EB9 - 14 modules in place.

Figure 2, above, shows a simplified location of forces being applied to the saddle in load case EB9, in which there are 14 modules in place that are only supported by the saddle. With only 14 modules in place, only 1 swivel bolt and the key are in contact. The reaction force, $\mathrm{F}$, is equal to 7 times the weight of a module. The saddle reaction forces, $\mathrm{F}_{\mathrm{r}}$ and $\mathrm{F}_{\mathrm{s}}$, can be easily solved by statics as shown below.

$$
\begin{array}{ll}
\Sigma F_{y} & F_{r} \cos \theta+F_{s} \sin \theta=F \\
\Sigma F_{x} & F_{r} \sin \theta=F_{s} \cos \theta \\
& F_{r}=F \cos \theta \\
& F_{s}=F \sin \theta
\end{array}
$$


However, the sum of the moments about the IP must be equal to zero in order to achieve equilibrium. Therefore,

$$
\Sigma M_{I P}-R F_{s}+X F=0
$$

Solving for $\mathrm{X}$, we find

$$
\begin{aligned}
& X=\frac{R F_{s}}{F} \\
& X=R \sin \theta .
\end{aligned}
$$

If the angle is

$$
\theta \approx 34^{0}
$$

then

$$
X \approx .56 R
$$

Using $R=4,173 \mathrm{~mm}$, we find that $X=2,333 \mathrm{~mm}$, which is not underneath the saddle support beam. If the support point is located at $3,000 \mathrm{~mm}$ at the center of the support beam, then an additional moment, [ $\mathrm{M}=(3000 \mathrm{~mm}-\mathrm{X})^{*} \mathrm{~F}$ ], is needed in order to achieve static equilibrium. In order to check this analysis, the saddle support beam in the FE model was restrained in load case EB9 along its edges in the vertical direction, so that it could support a reaction moment. The reaction forces were then examined and the equivalent location of the reaction force was found. This is plotted below and shows that the reaction force would have to be applied outside of the beam to achieve equilibrium. The location of the reaction force is in reasonable agreement with the location of the reaction force calculated in the simplified hand calculations discussed above. 


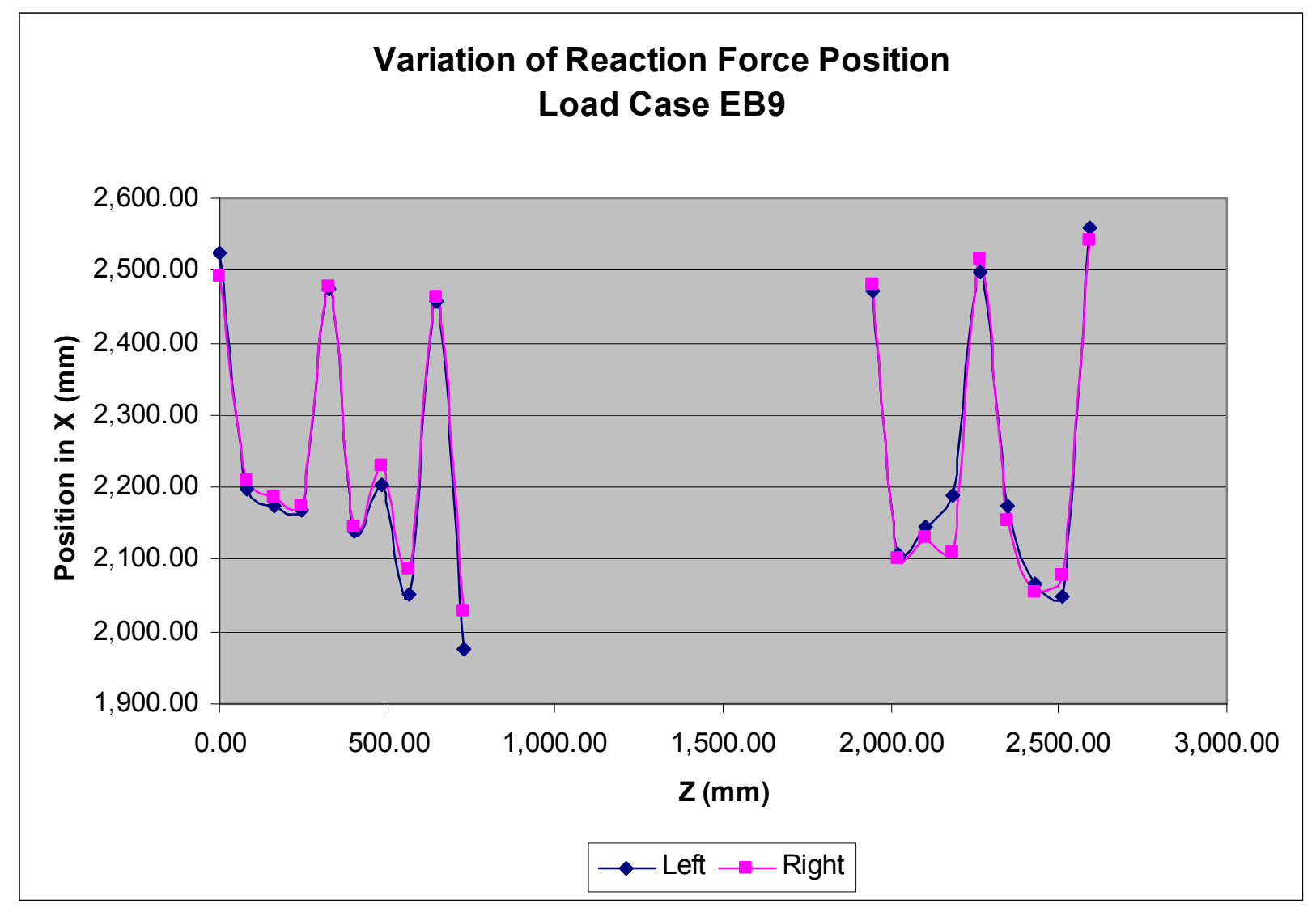

Fig. 3. Variation of reaction force position--load case EB9.

In order to achieve equilibrium during assembly, the support cradle, which is used to initially assemble the first 12 modules, will have to be kept in place. In this case, the support cradle carries the entire load of the modules and only a minimal load is applied to the saddles. This analysis is shown in load case EB10 where there are 14 modules in place supported by a combination of the support cradle and the saddles. The forces acting on the saddles in this load case are virtually zero.

The same analysis and conclusions apply to load case EB7, in which there are 24 modules in place and the cryostat load is applied. The forces acting on the saddle in the back of this load case are shown in Fig. 4 below. Static equilibrium can be found using the following equations.

$$
\begin{array}{ll}
\Sigma F_{y} \quad & F_{1} \cos \theta+F_{2} \cos \alpha+F_{s} \sin \theta-F_{c}=F \\
& F=W+F_{c} \\
& F_{1} \cos \theta+F_{2} \cos \alpha+F_{s} \sin \theta=W \\
\Sigma F_{x} \quad & F_{1} \sin \theta+F_{2} \sin \alpha-F_{s} \cos \theta=0.0
\end{array}
$$




$$
\begin{gathered}
\Sigma M_{o} \quad-R F_{s}+X F-A F_{c}=0.0 \\
F_{s}=\frac{X F-A F_{c}}{R}
\end{gathered}
$$

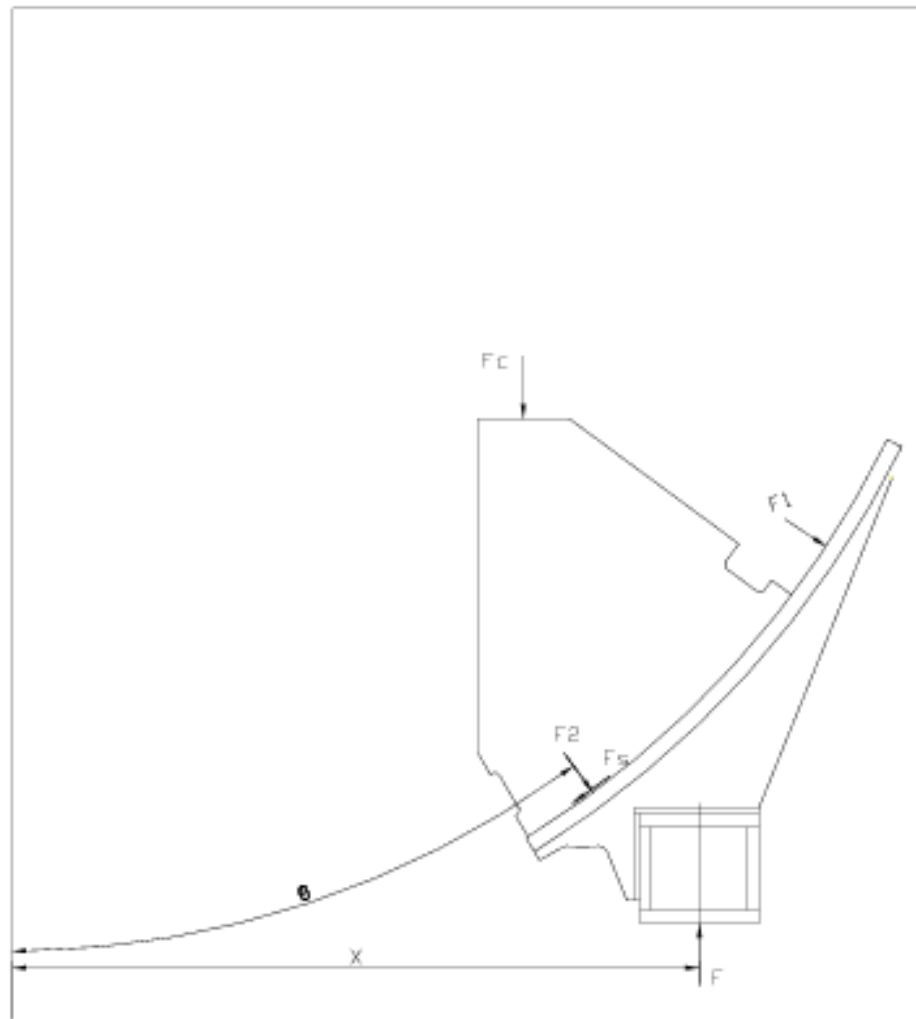

Fig. 4. Static analysis of load case EB7 - 24 modules in place with the cryostat load applied.

Solving for $\mathrm{F}_{\mathrm{r} 1}, \mathrm{~F}_{\mathrm{r} 2}$, and $\mathrm{F}_{\mathrm{s}}$ results in swivel bolt forces, which are in tension. However, the swivel bolt forces must be in compression. This can only be achieved by either moving the location of the support point, $\mathrm{X}$, or by adding the force, $\mathrm{P}$, as shown in the figure below. The force, $\mathrm{P}$, can be added by placing a compression beam between the back cryostat supports. The static equilibrium equations in this case are

$$
\begin{aligned}
\Sigma F_{y} \quad & F_{1} \cos \theta+F_{2} \cos \alpha+F_{s} \sin \theta-F_{c}=F \\
& F=W+F_{c} \\
& F_{1} \cos \theta+F_{2} \cos \alpha+F_{s} \sin \theta=W \\
\Sigma F_{x} \quad & F_{1} \sin \theta+F_{2} \sin \alpha-F_{s} \cos \theta=-P
\end{aligned}
$$




$$
\begin{gathered}
\Sigma M_{o} \quad-R F_{s}+X F-A F_{c}+B P=0.0 \\
F_{s}=\frac{X F-A F_{c}}{R}+\left(\frac{B}{R}\right) P .
\end{gathered}
$$

If

$$
\mathrm{P}=142 \text { tons, }
$$

then

$$
F_{s}=0.0 .
$$

Solving for $\mathrm{P}, \mathrm{F}_{\mathrm{r} 1}, \mathrm{~F}_{\mathrm{r} 2}$, and $\mathrm{F}_{\mathrm{s}}$ results in positive value of the swivel bolt forces. These four unknowns were solved with only 3 equations by iterating the value of $P$ from zero to a final value of 18 tons, at which point the swivel bolt forces became positive. The value of 41 tons for $\mathrm{P}$ is the minimum value, which is needed to have the swivel bolts in compression. The output of the FORTRAN program that solved this problem can be found in Appendix 1 and shows the forces on the back saddle for every module during the assembly. In order to check this static analysis, a truss element was placed in the FE model between the back cryostat supports. The resulting values of the forces on the saddle are compared below, between the FE model and the static analysis. The static analysis compares well with the FE model considering the large deflections of the saddle ( $3 \mathrm{~mm})$ at the top swivel bolt and that the force, F1, in the FE model is actually a sum of the forces acting on 3 different swivel bolts at 3 different module positions.

\begin{tabular}{|c|c|c|c|c|}
\hline & P (tons) & F1 (tons) & F2 (tons) & Fs (tons) \\
\hline Static Analysis & 41 & 32 & 0 & 78 \\
\hline FE Model & 34 & 19 & 0 & 65 \\
\hline$\%$ Difference & $17 \%$ & $40 \%$ & $0 \%$ & $16 \%$ \\
\hline
\end{tabular}

A compression member between the back cryostat supports is needed in order to generate the force, $\mathrm{P}$. This compression member will have to be similar to a turnbuckle so that the value of $\mathrm{P}$ can be varied. In order to remove the compression member after the EB assembly is stable, it will be necessary to reduce the force on it to zero.

Finally, a static analysis identical to the one described above was carried out for the addition of every module to the assembly from 14 modules in place to 64 modules in place. This analysis shows that with 14, 15, 16 and 24, 25, 26, 27, 28 modules in place, the assembly is unstable, because an additional moment is needed at the bottom of the saddle in order to achieve stability. In all of the load cases, additional fixturing will be needed in order to provide the necessary support. 


\section{Forces between Modules}

The charts below show the forces between the modules for the load cases that have been examined. It is clear from these charts that the forces decline with the number of modules, which are assembled and are the maximum when the entire EB has been assembled.

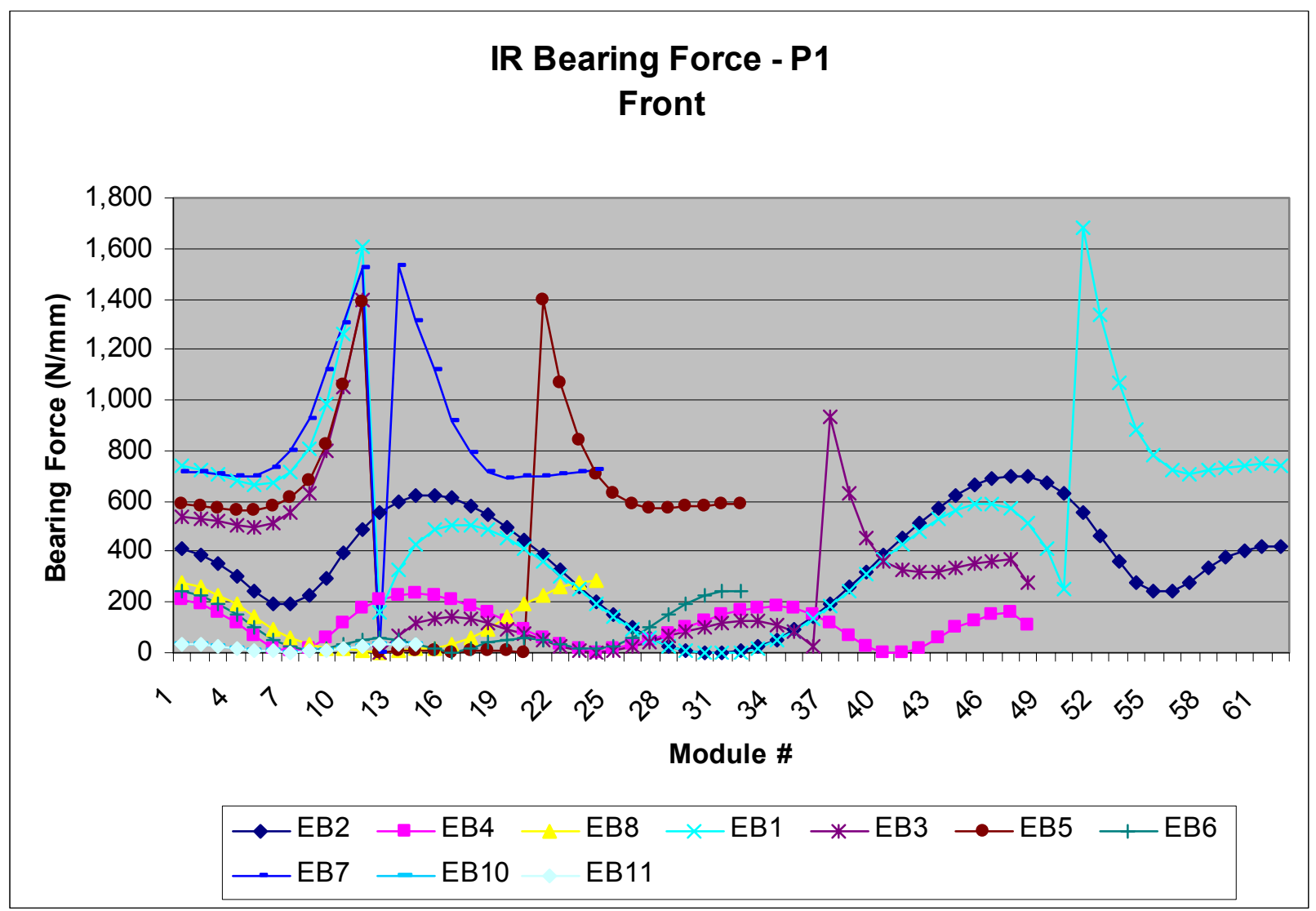



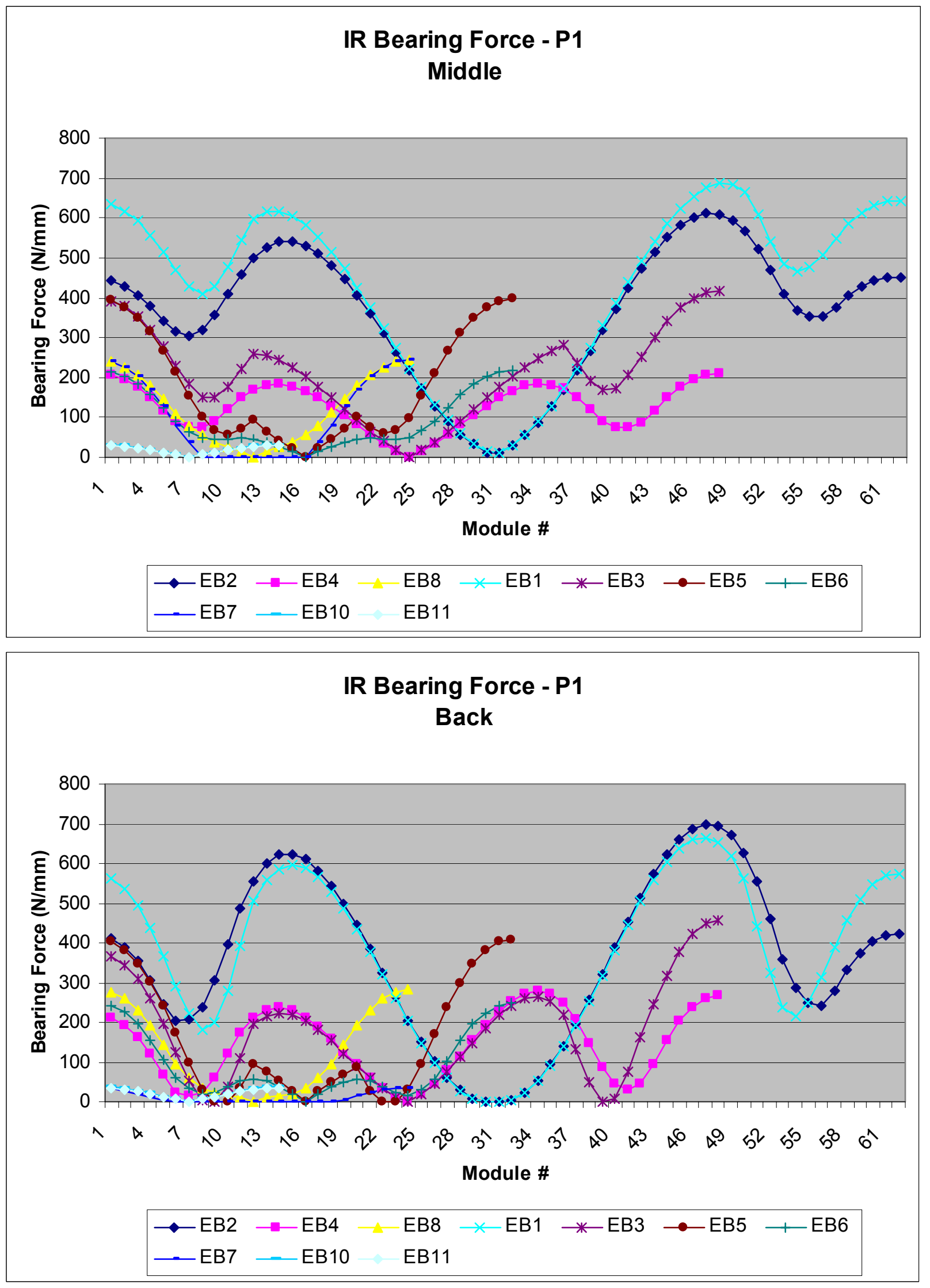


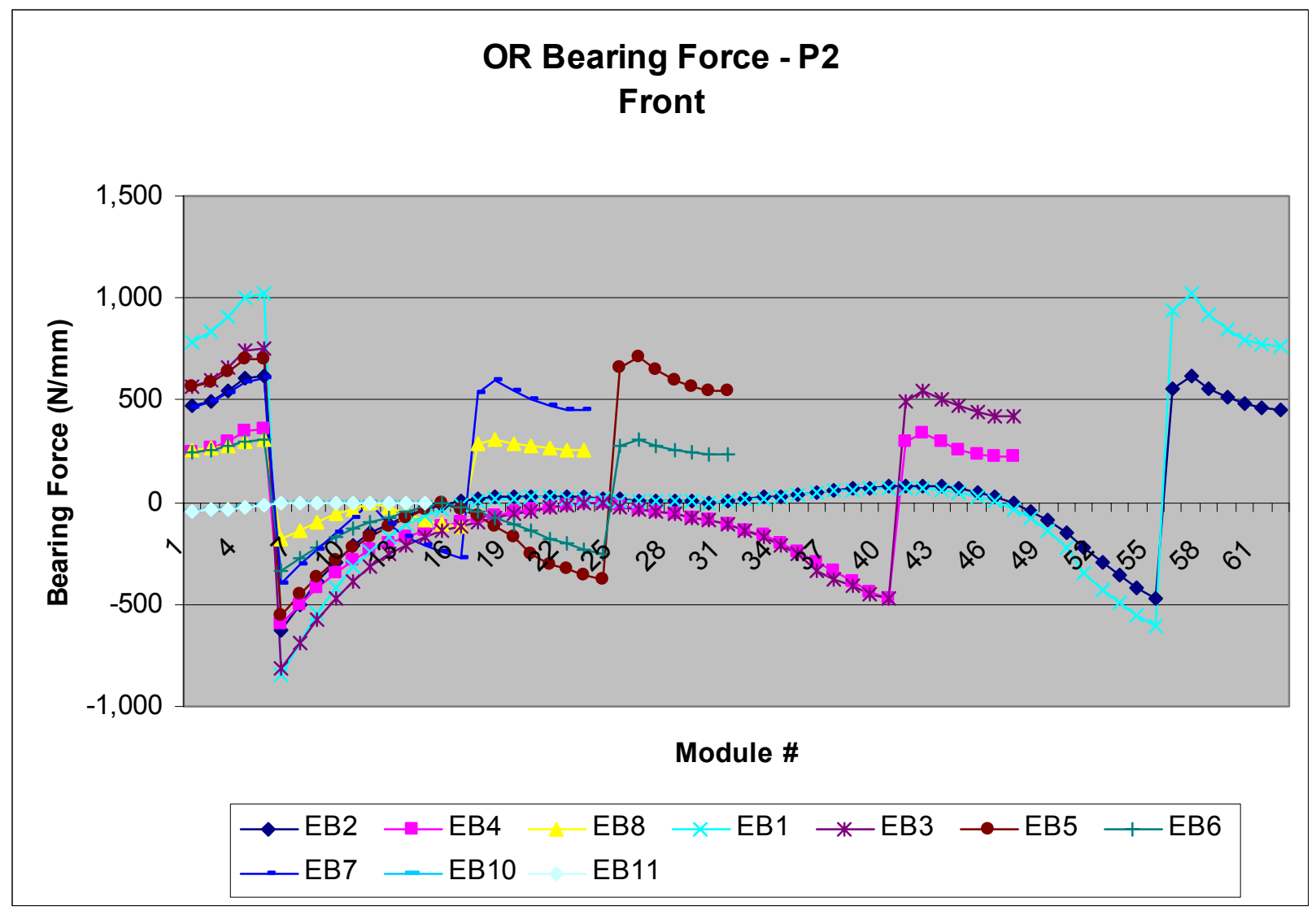



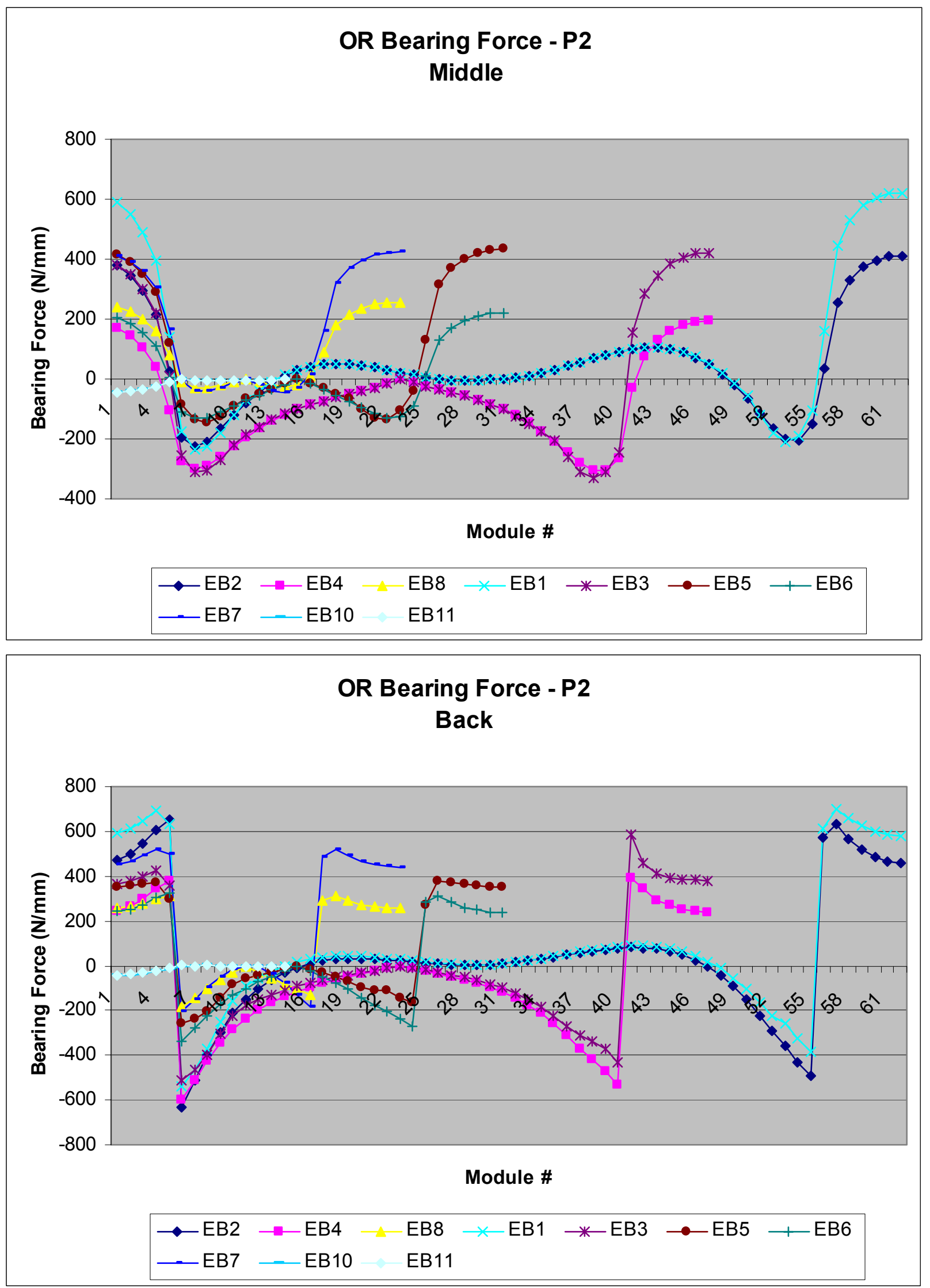


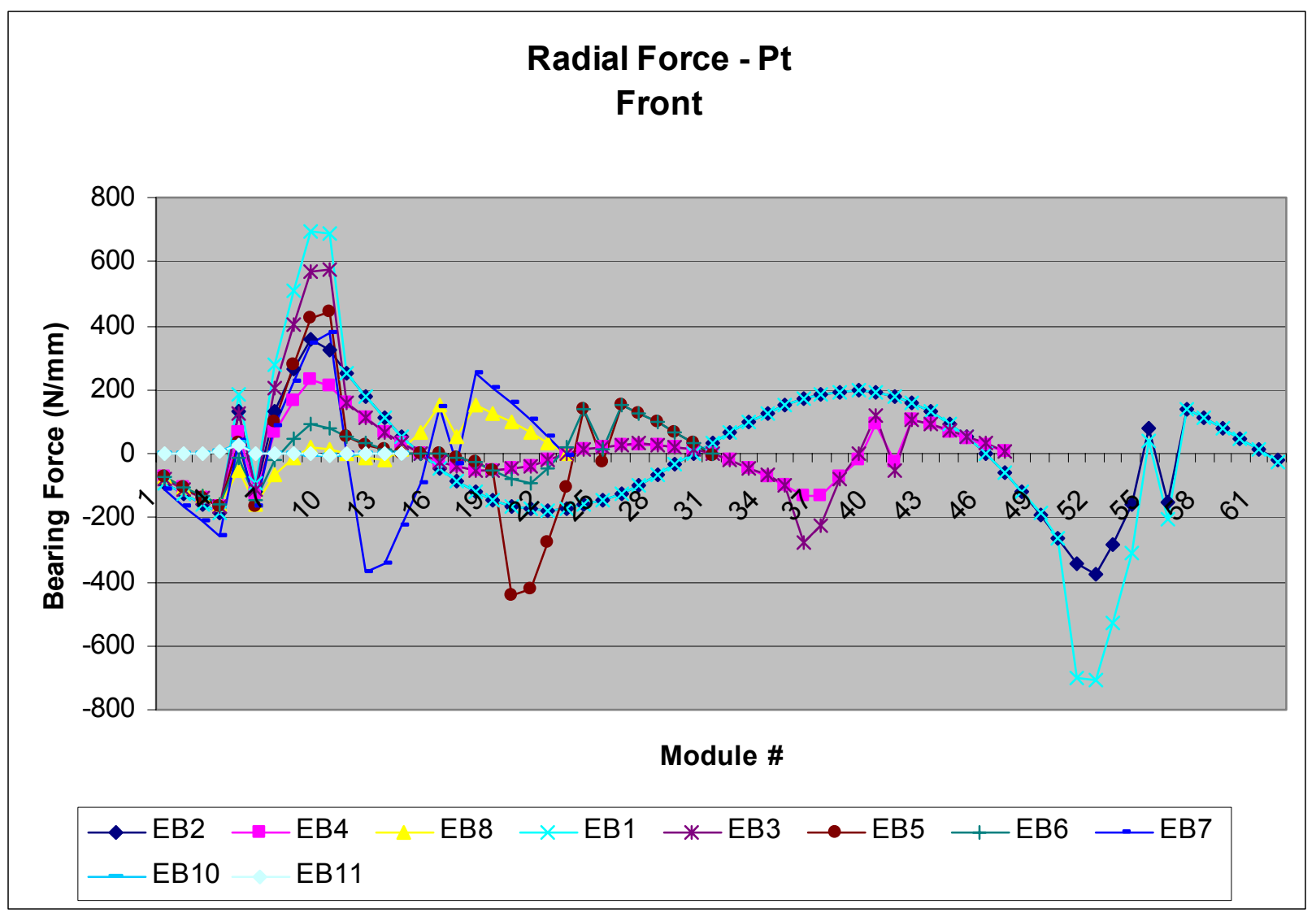




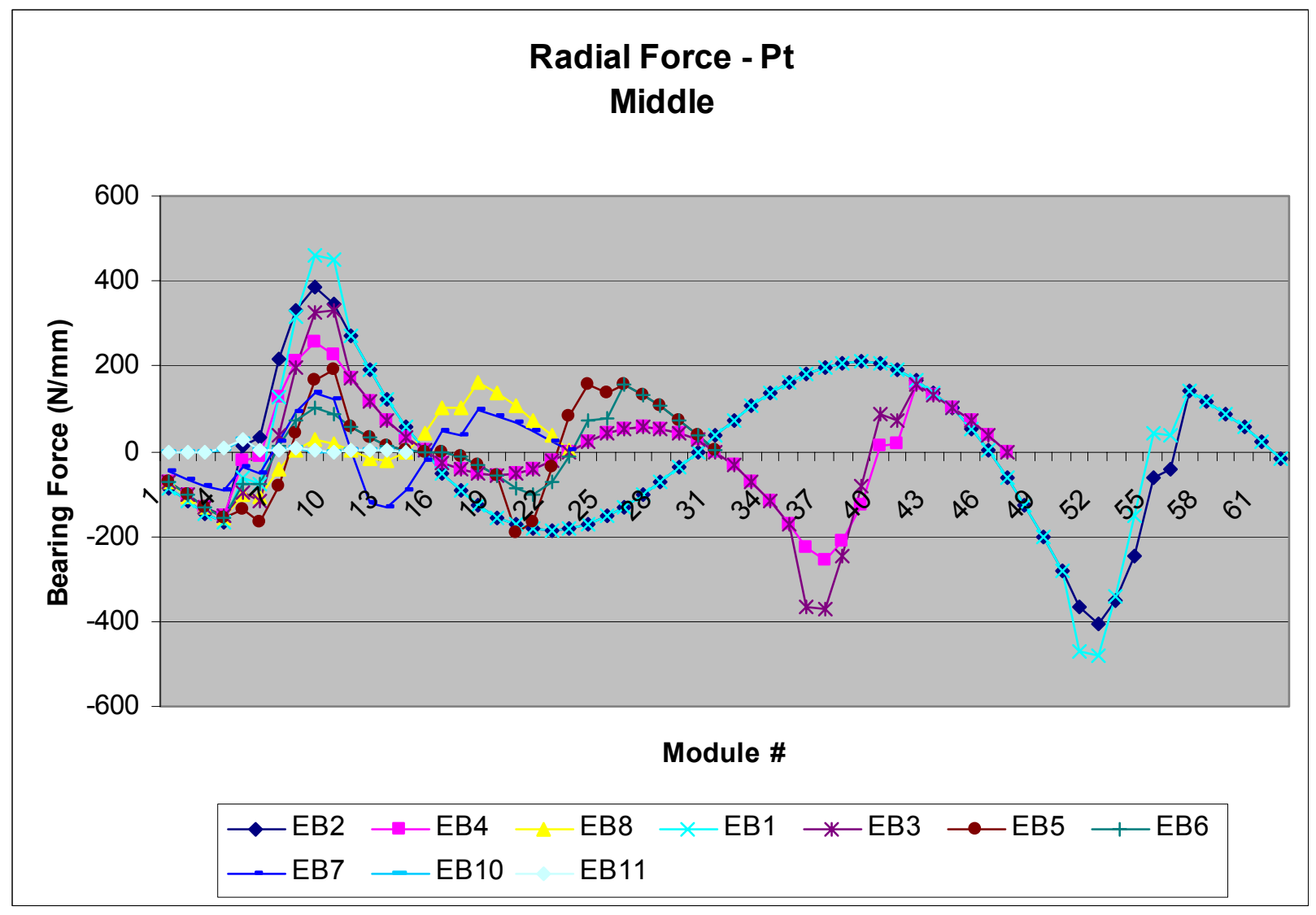




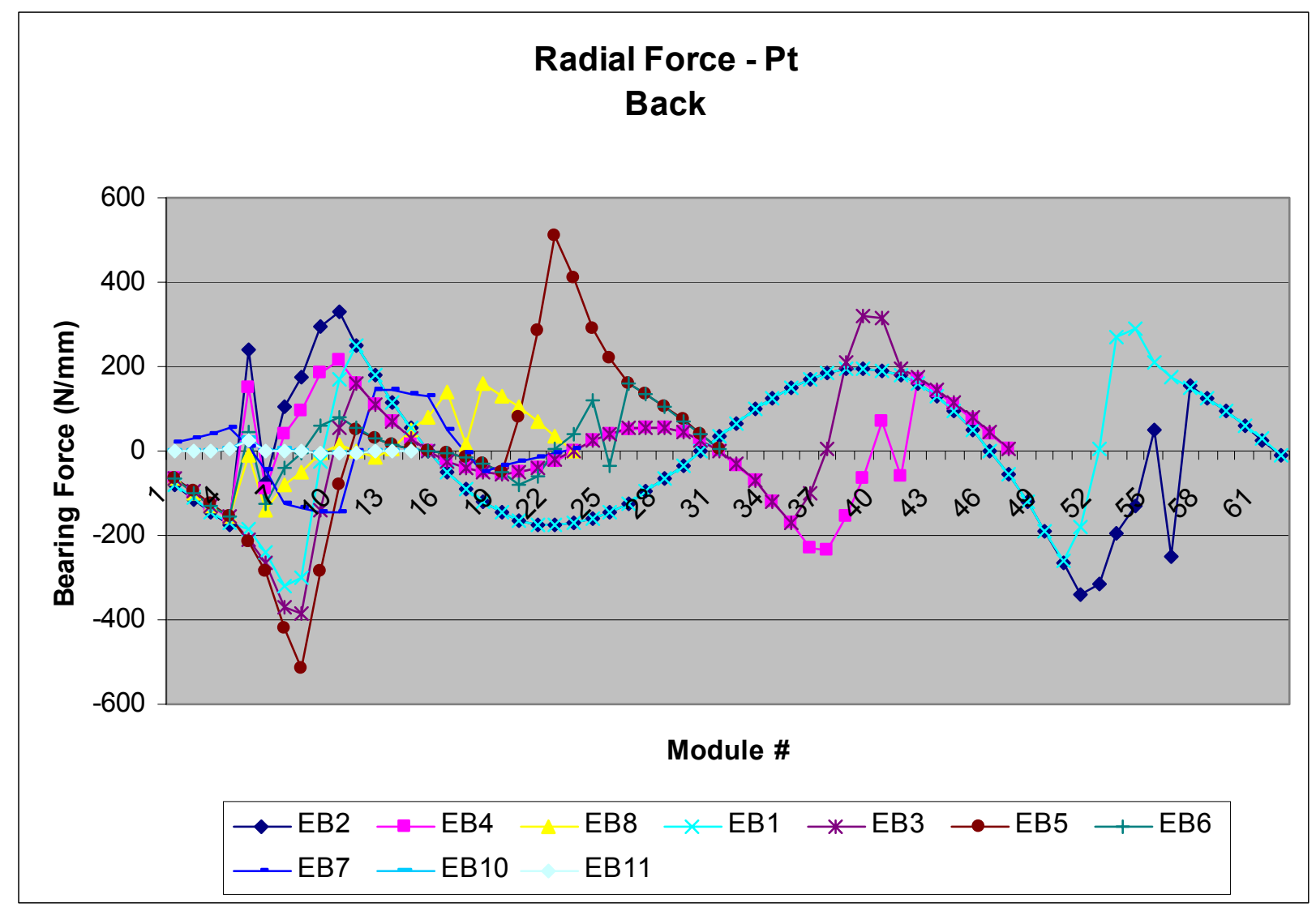

\section{Saddle Forces}

The force on the saddles from the EB also changes as the modules are assembled. These are shown in tables below. Once again, the maximum loads on the saddle occur when the entire EB has been assembled. There are three special cases. The first is load case EB7, in which there are 24 modules in place and the cryostat load is first applied. This load case, as discussed above, is unstable unless a compression member is placed between the back cryostat supports. The results below reflect an FE model, in which a truss is placed between the back cryostat supports, and the resulting compression force on this truss is $\mathrm{XXX}$ tons.

The results for load case 9, 14 modules supported only by the saddle, are not shown, because this case, as discussed above, is unstable. The results for load case 10, which is 14 modules in place supported by the assembly cradle and saddles, are not shown, because the forces on the saddles are nearly zero. 


\section{Load Case EB1}

\begin{tabular}{cccccccc} 
& \multicolumn{8}{c}{ Radial Forces } \\
Z1 & M6 & M8 & M9 & M10 & M11 & M12 & Forces \\
M7 \\
Z2 & 110,500 & 16,250 & 115,500 & 227,500 & 128,000 & 0 & $-354,600$ \\
Z3 & 236,900 & 345,400 & 209,500 & 115,700 & 41,490 & 0 & $-740,800$ \\
Z4 & 114,100 & 216,300 & 194,100 & 188,500 & 35,540 & 0 & $-996,500$ \\
& & & & 188,900 & 88,190 & 0 & $-595,200$
\end{tabular}

\section{Load Case EB2}

\begin{tabular}{cccccccc} 
& \multicolumn{8}{c}{ Radial Forces } & & Key \\
Z1 & M6 & M8 & M9 & M10 & M11 & M12 & Forces \\
M7 & 202,100 & 136,600 & 97,690 & 118,400 & 58,260 & 0 & $-426,700$ \\
Z2 & 148,800 & 220,800 & 160,700 & 95,170 & 18,520 & 0 & $-618,900$ \\
Z3 & 208,800 & 249,800 & 181,000 & 123,900 & 30,590 & 0 & $-653,800$ \\
Z4 & 139,600 & 155,900 & 102,300 & 84,700 & 27,270 & 0 & $-403,600$
\end{tabular}

\section{Load Case EB3}

\begin{tabular}{cccccccc} 
& \multicolumn{8}{c}{ Radial Forces } & & Key \\
Z1 & M6 & M8 & M9 & M10 & M11 & M12 & M7 \\
Z1 & 0 & 0 & 83,900 & 201,000 & 117,400 & 0 & $-251,300$ \\
Z2 & 32,700 & 169,000 & 169,000 & 93,580 & 37,500 & 0 & $-594,500$ \\
Z3 & 187,000 & 287,200 & 225,000 & 159,700 & 28,670 & 0 & $-841,300$ \\
Z4 & 85,490 & 181,900 & 169,800 & 169,300 & 82,360 & 0 & $-500,390$
\end{tabular}

\section{Load Case EB4}

\begin{tabular}{|c|c|c|c|c|c|c|c|}
\hline & M6 & M8 & $\begin{array}{l}\text { Radial } \\
\text { M9 }\end{array}$ & $\begin{array}{r}\text { orces } \\
\text { M10 }\end{array}$ & M11 & M12 & Forces \\
\hline $\mathrm{Z1}$ & 154,100 & 104,100 & 74,580 & 90,210 & 44,620 & 0 & $-326,500$ \\
\hline $\mathbf{Z 2}$ & 113,800 & 168,700 & 122,900 & 72,340 & 14,140 & 0 & $-471,470$ \\
\hline Z3 & 159,700 & 190,800 & 138,300 & 94,240 & 23,360 & 0 & $-498,000$ \\
\hline Z4 & 106,400 & 118,900 & 78,060 & 64,340 & 20,820 & 0 & $-308,700$ \\
\hline
\end{tabular}




\section{Load Case EB5}

\begin{tabular}{cccccccc} 
& \multicolumn{8}{c}{ Radial Forces } & & Key \\
& M6 & M8 & M9 & M10 & M11 & M12 & M7 \\
Z1 & 0 & 0 & 34,900 & 178,800 & 110,800 & 533 & $-142,900$ \\
$\mathbf{Z 2}$ & 0 & 13,480 & 123,200 & 77,770 & 35,620 & 1,186 & $-427,700$ \\
$\mathbf{Z 3}$ & 117,400 & 217,000 & 179,800 & 131,500 & 22,620 & 0 & $-680,000$ \\
$\mathbf{Z 4}$ & 60,450 & 149,800 & 146,900 & 152,600 & 78,240 & 0 & $-399,400$
\end{tabular}

\section{Load Case EB6}

\begin{tabular}{cccccccc} 
& \multicolumn{7}{c}{ Radial Forces } \\
Z1 & M6 & M8 & M9 & M10 & M11 & M12 & $\begin{array}{c}\text { Key } \\
\text { Forces }\end{array}$ \\
M7 \\
Z2 & 762,600 & 69,420 & 50,450 & 60,150 & 29,630 & 0 & $-219,700$ \\
Z3 & 106,800 & 112,300 & 82,520 & 47,940 & 9,137 & 0 & $-312,700$ \\
Z4 & 70,880 & 79,370 & 52,750 & 42,780 & 13,640 & 0 & $-207,700$
\end{tabular}

\section{Load Case EB7}

\begin{tabular}{|c|c|c|c|c|c|c|c|}
\hline \multicolumn{7}{|c|}{ Radial Forces } & $\begin{array}{c}\text { Key } \\
\text { Forces }\end{array}$ \\
\hline & M6 & M8 & M9 & M10 & M11 & M12 & M7 \\
\hline Z1 & 111,400 & 127,700 & 97,720 & 94,390 & 56,720 & 0 & $-323,700$ \\
\hline $\mathbf{Z 2}$ & 142,800 & 198,000 & 149,800 & 102,600 & 18,110 & 0 & $-536,630$ \\
\hline $\mathrm{Z3}$ & 0 & 15,730 & 83,430 & 68,540 & 23,290 & 0 & $-424,500$ \\
\hline $\mathrm{Z4}$ & 0 & 0 & 0 & 0 & 0 & 0 & $-216,900$ \\
\hline
\end{tabular}

\section{Load Case EB8}

\begin{tabular}{cccccccc} 
& \multicolumn{7}{c}{ Radial Forces } \\
& M6 & M8 & M9 & M10 & M11 & M12 & Forces \\
M1 \\
Z1 & 76,630 & 52,100 & 38,420 & 45,070 & 22,020 & 0 & 165,900 \\
Z2 & 57,000 & 84,080 & 62,350 & 35,750 & 6,578 & 0 & 233,330 \\
Z3 & 80,110 & 95,160 & 70,070 & 46,690 & 11,160 & 0 & 246,600 \\
Z4 & 52,990 & 59,620 & 40,100 & 31,970 & 9,969 & 0 & 156,900
\end{tabular}

\section{Deflections}

The deflections in the vertical direction are shown below. It should be noted that in the load cases with the cryostat there are significant differences between the front and back of the EB. 
The deflections at the cryostat support points are shown for each load case in the table below:

\begin{tabular}{|c|c|c|c|c|}
\hline \multirow{2}{*}{ Load Case } & \multicolumn{2}{|c|}{$\begin{array}{c}\text { Front Cryostat } \\
\text { Support }\end{array}$} & \multicolumn{2}{c|}{$\begin{array}{c}\text { Back Cryostat } \\
\text { Support }\end{array}$} \\
\cline { 2 - 5 } & X(mm) & Y (mm) & X (mm) & Y (mm) \\
\hline EB1 & -1.7 & -3.3 & 2.2 & -2.1 \\
\hline EB2 & -0.8 & -2.0 & -0.4 & -0.4 \\
\hline EB3 & -1.5 & -2.7 & 2.8 & -2.1 \\
\hline EB4 & -0.5 & -1.4 & -0.2 & -0.3 \\
\hline EB5 & -0.8 & -2.1 & 3.6 & -2.2 \\
\hline EB6 & 0 & -0.8 & 0.3 & -0.4 \\
\hline EB7 & -0.6 & -1.7 & 1.1 & -1.3 \\
\hline EB8 & 0.4 & -0.5 & 0.5 & -0.4 \\
\hline
\end{tabular}

* positive $\mathrm{X}$ values are deflections toward the center of the EB.

Deflections at the Top of the Saddle

\begin{tabular}{|c|c|c|c|c|c|c|c|c|}
\hline \multirow{2}{*}{$\begin{array}{l}\text { Load } \\
\text { Case }\end{array}$} & \multicolumn{2}{|c|}{$\mathrm{Z1}$} & \multicolumn{2}{|c|}{$\mathbf{Z 2}$} & \multicolumn{2}{|c|}{$\mathbf{Z 3}$} & \multicolumn{2}{|c|}{$\mathbf{Z 4}$} \\
\hline & $\mathrm{X}(\mathrm{mm})$ & $Y(\mathrm{~mm})$ & $\mathrm{X}(\mathrm{mm})$ & $\mathrm{Y}(\mathrm{mm})$ & $\mathrm{X}(\mathrm{mm})$ & $Y(\mathrm{~mm})$ & $\mathrm{X}(\mathrm{mm})$ & Y (mm) \\
\hline EB1 & -1.9 & -2.5 & -1.4 & -2.0 & -0.2 & -1.0 & 1.1 & -0.7 \\
\hline EB2 & -0.9 & -1.6 & -1.0 & -1.4 & -0.9 & -1.2 & -0.8 & -1.1 \\
\hline EB3 & -1.5 & -2.1 & -1.0 & -1.6 & 0.6 & -0.7 & -1.5 & -0.4 \\
\hline EB4 & -0.6 & -1.1 & -0.6 & -1.0 & -0.6 & -0.9 & -0.5 & -0.8 \\
\hline EB5 & -0.9 & -1.6 & -0.3 & -1.1 & 1.3 & -0.2 & 2.2 & -0.1 \\
\hline EB6 & 0.0 & -0.6 & 0.0 & -0.5 & 0.0 & -0.4 & 0.0 & -0.4 \\
\hline EB7 & -0.7 & -1.2 & -0.4 & -0.9 & 0.7 & -0.3 & 0.9 & -0.4 \\
\hline EB8 & 0.3 & -0.3 & 0.2 & -0.3 & 0.3 & -0.2 & 0.3 & -0.2 \\
\hline
\end{tabular}

Deflections at the Bottom of the Saddle

\begin{tabular}{|c|c|c|c|c|c|c|c|c|}
\hline \multirow{2}{*}{$\begin{array}{c}\text { Load } \\
\text { Case }\end{array}$} & \multicolumn{2}{|c|}{ Z1 } & \multicolumn{2}{c|}{ Z2 } & \multicolumn{2}{c|}{ Z3 } & \multicolumn{2}{c|}{ Z4 } \\
\cline { 2 - 9 } EB1 & 0.0 & -1.3 & 0.0 & -1.2 & 0.2 & -1.3 & 0.2 & -1.7 \\
\hline EB2 & 0.0 & -1.0 & 0.0 & -0.8 & -0.1 & -0.6 & 0.0 & -0.4 \\
\hline EB3 & 0.0 & -1.1 & 0.0 & -1.0 & 0.3 & -1.2 & 0.3 & -1.7 \\
\hline EB4 & 0.0 & -0.8 & 0.0 & -0.6 & 0.0 & -0.5 & 0.0 & -0.4 \\
\hline EB5 & 0.0 & -1.0 & 0.2 & -0.9 & 0.4 & -1.2 & 0.3 & -1.8 \\
\hline EB6 & 0.0 & -0.6 & 0.0 & -0.5 & 0.0 & -0.5 & 0.1 & -0.4 \\
\hline EB7 & 0.0 & -0.9 & 0.0 & -0.7 & 0.2 & -0.8 & 0.2 & -1.1 \\
\hline EB8 & 0.1 & -0.6 & 0.1 & -0.5 & 0.1 & -0.4 & 0.1 & -0.4 \\
\hline
\end{tabular}




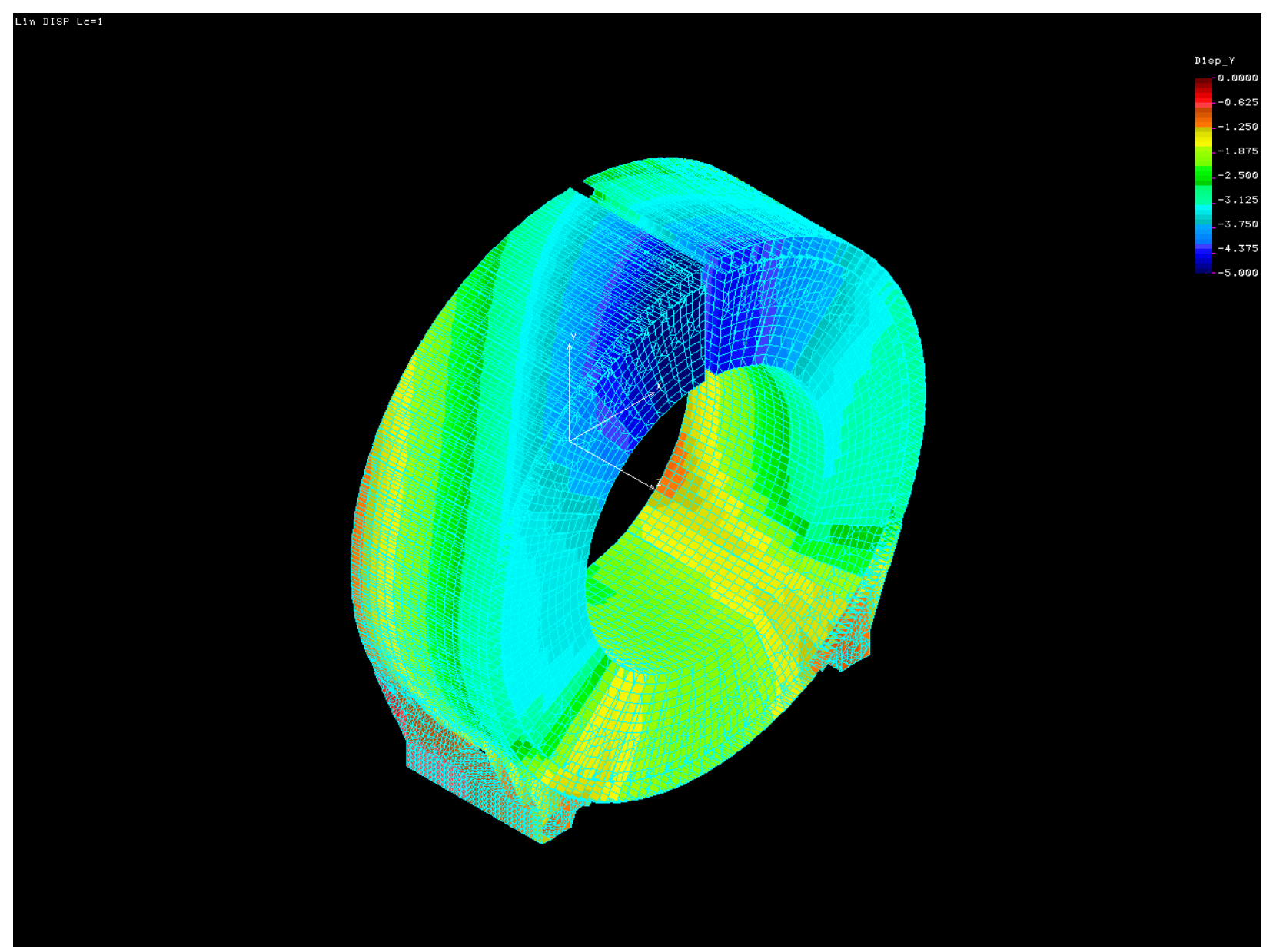

Vertical Deflections (mm)

Load Case EB1 
$8 / 9 / 2002$

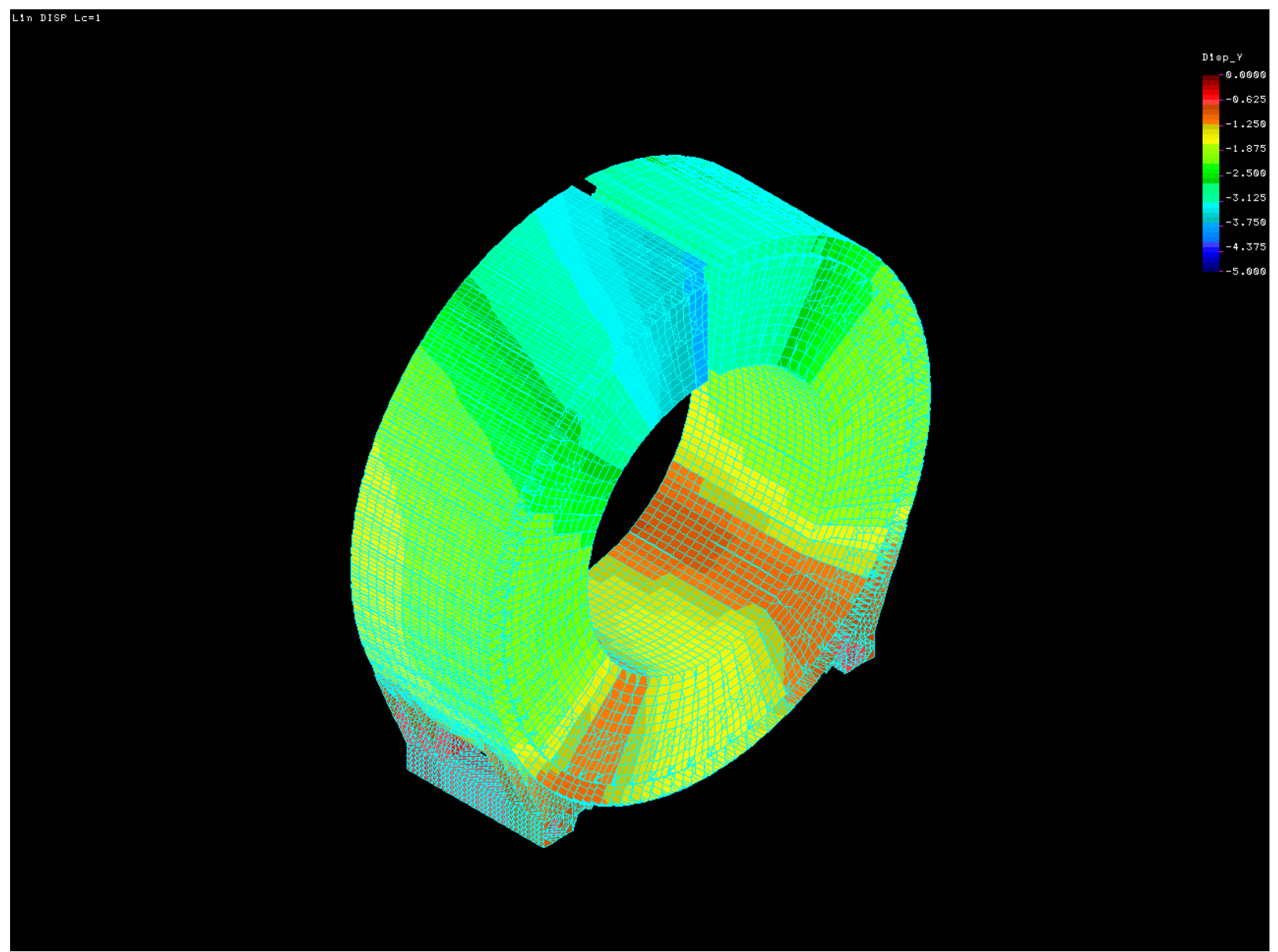

Vertical Deflections (mm)

Load Case EB2 
$8 / 9 / 2002$

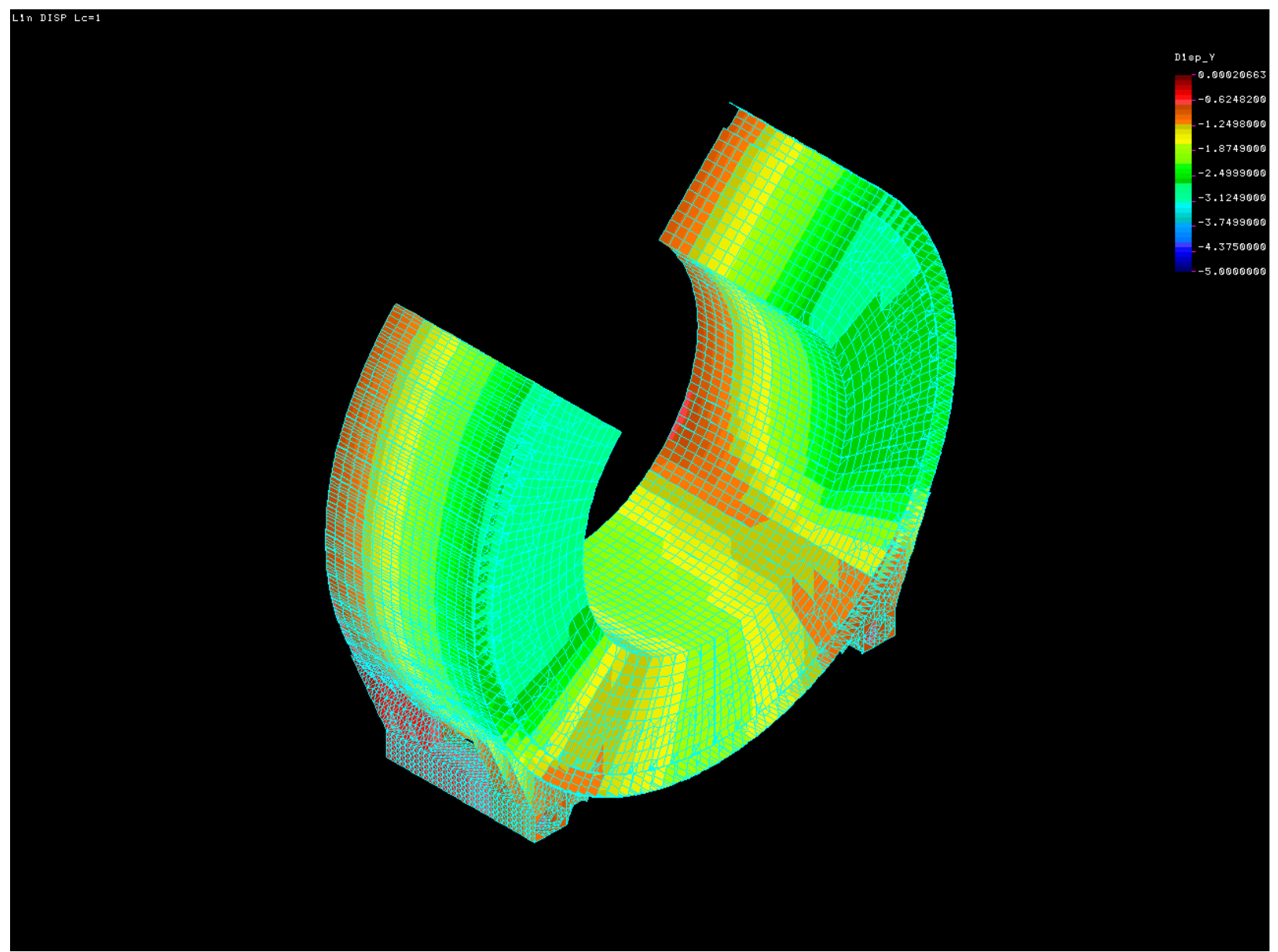

Vertical Deflections (mm)

Load Case EB3 
$8 / 9 / 2002$

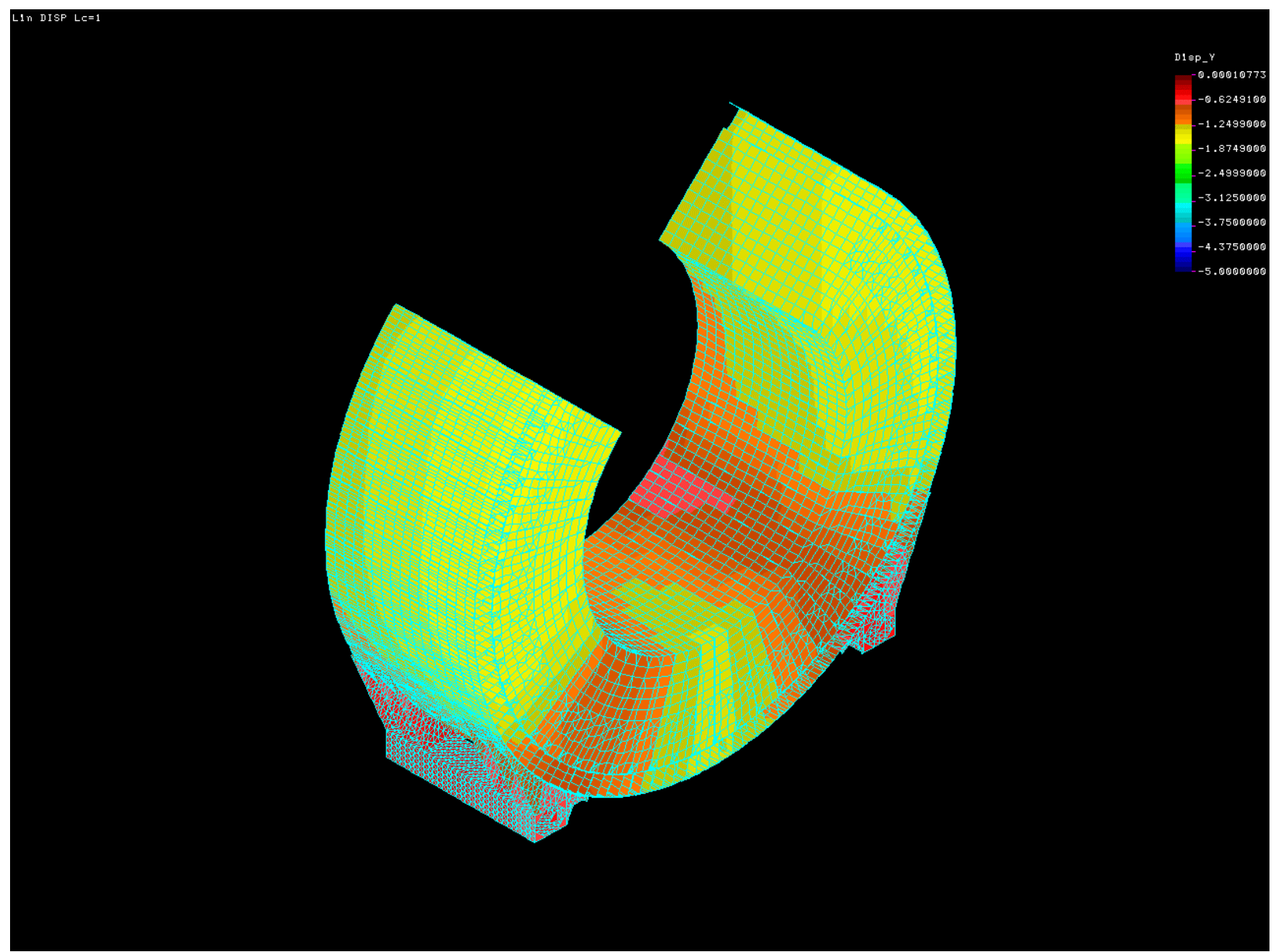

Vertical Deflections (mm)

Load Case EB4 
$8 / 9 / 2002$

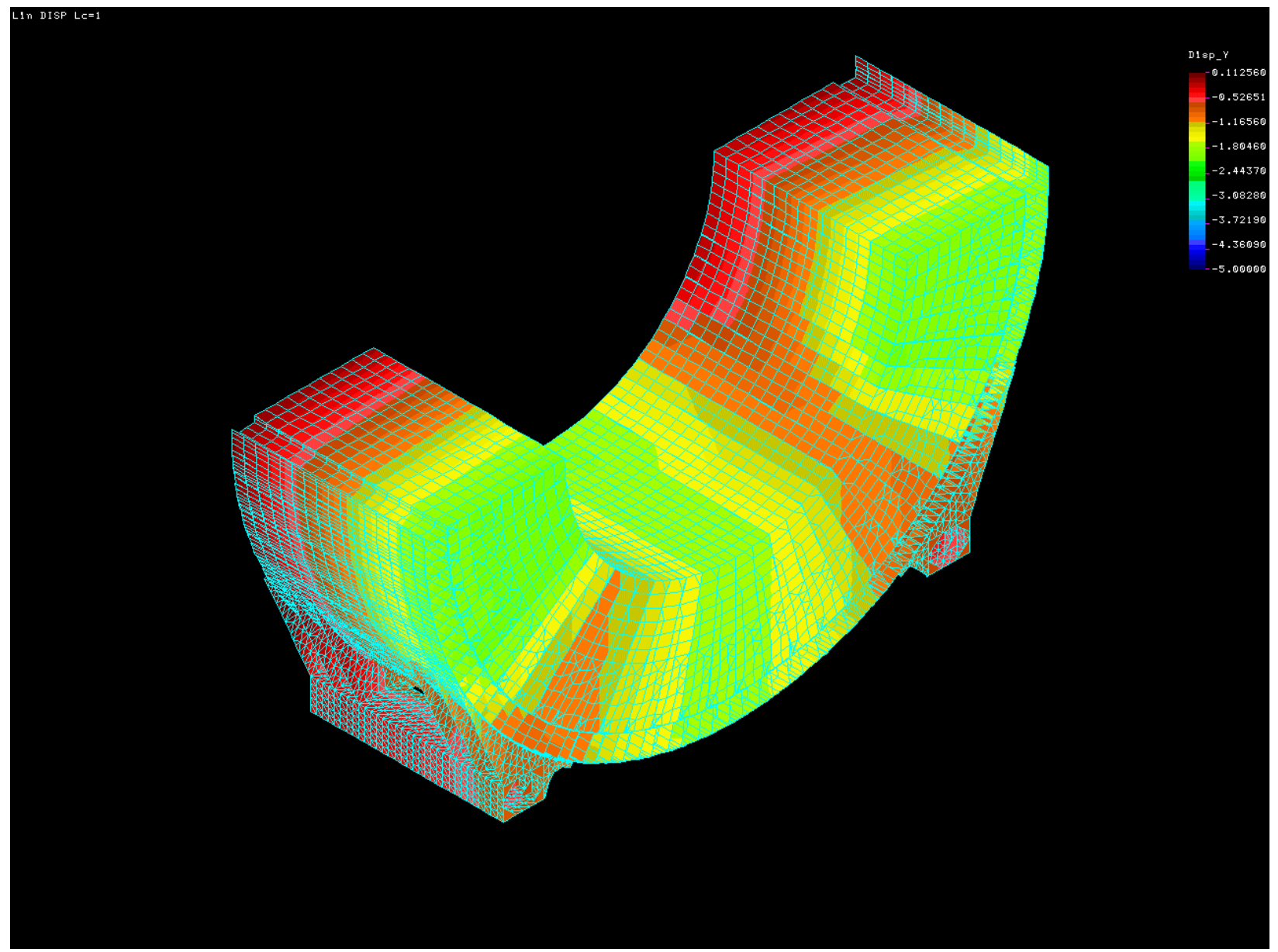

Vertical Deflections (mm)

Load Case EB5 
$8 / 9 / 2002$

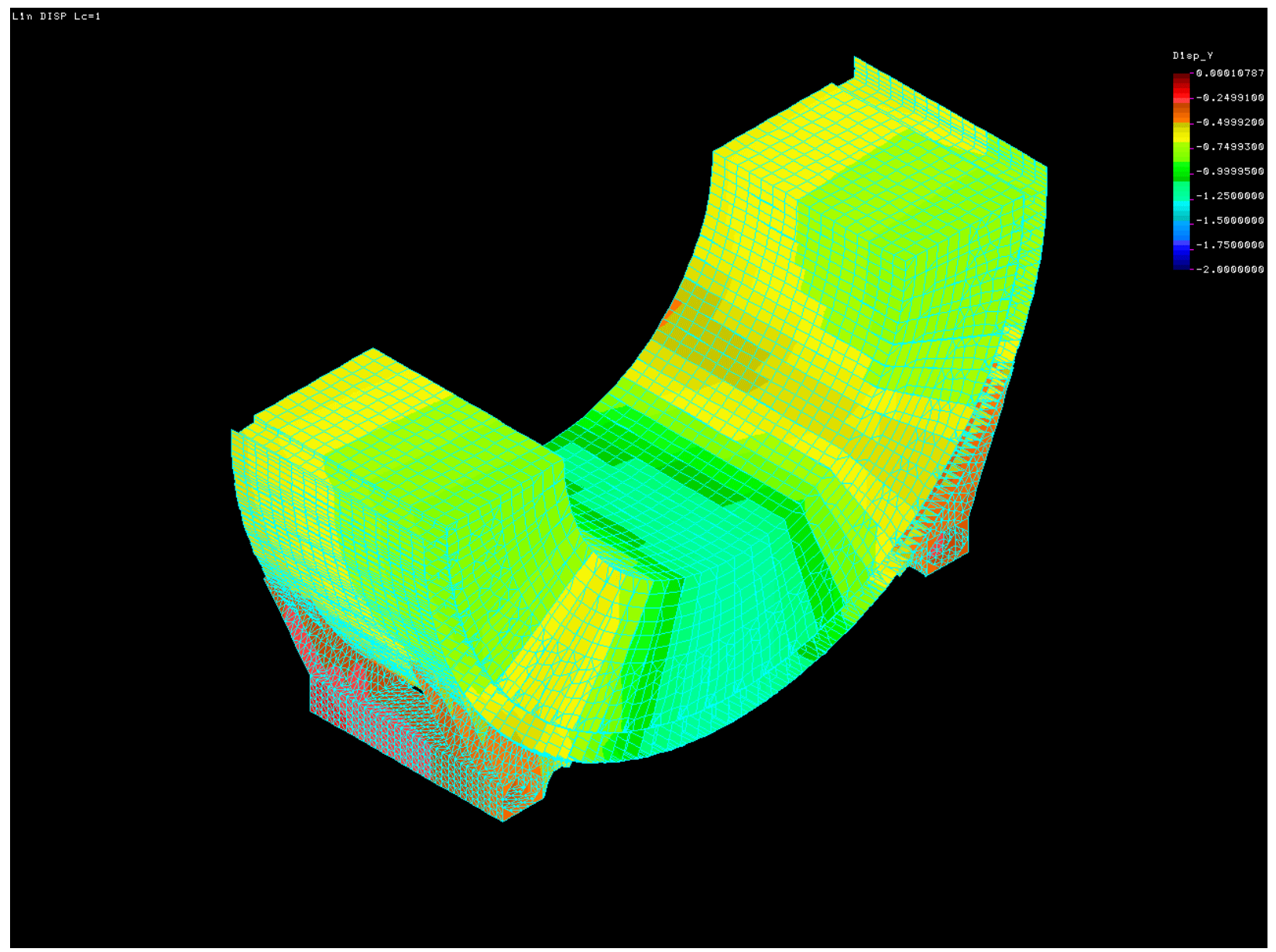

Vertical Deflections (mm)

Load Case EB6 


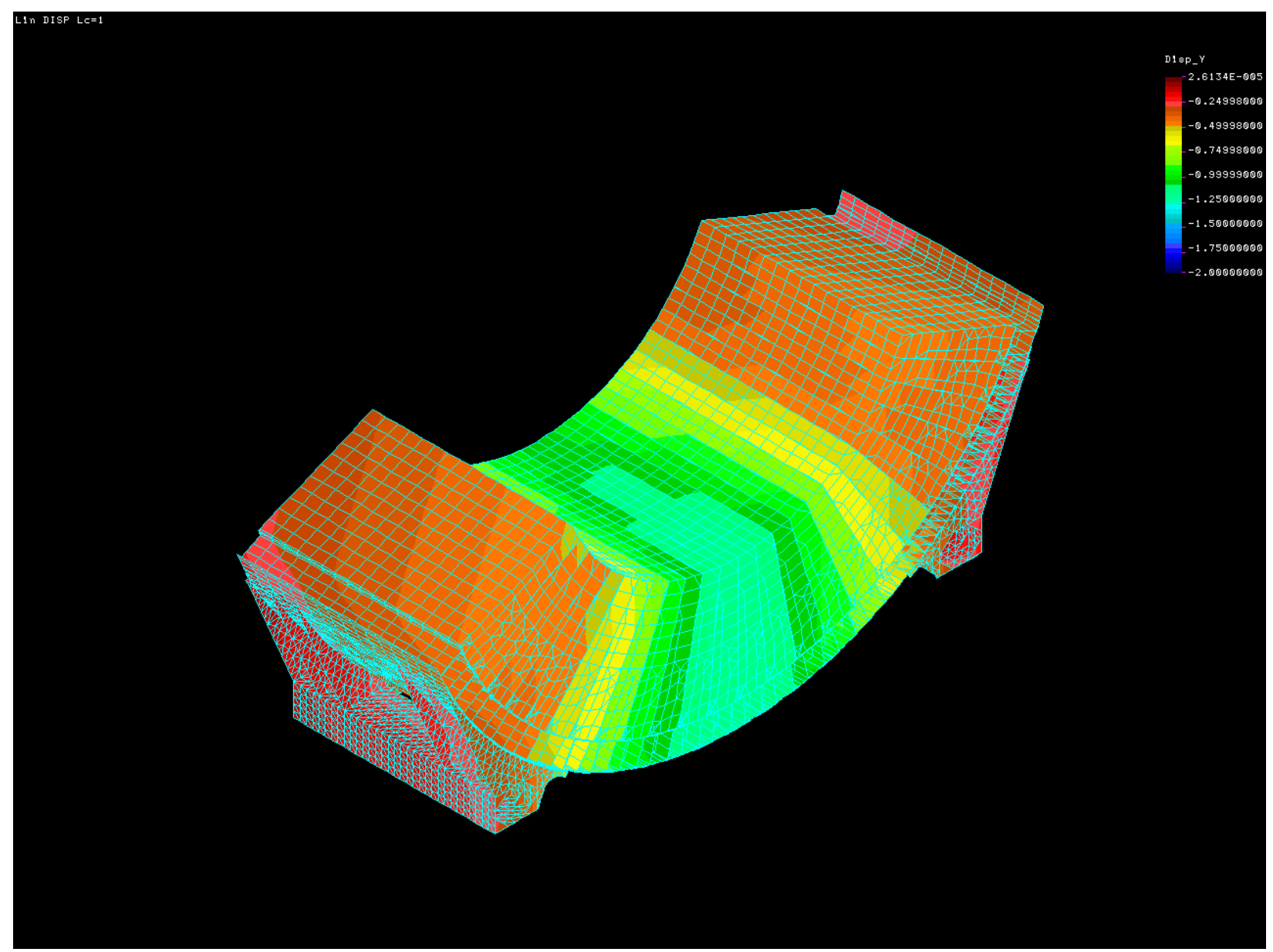

\section{Vertical Deflections (mm) \\ Load Case EB8}

\section{Conclusions}

The forces acting on the modules and support saddle during assembly for the load cases examined are all less than those found in the completed EB and far less than those used to design the EB.

- The EB assembly is not stable when there are 24 modules in place and the cryostat load is first applied. In order to stabilize the assembly, a stiffening beam is needed between the back cryostat supports. This stiffening beam is subjected to 34 tons. A static analysis shows that up to module 28 , the stiffening beam will be needed.

- The EB assembly is not stable when there are 14 modules and the saddle is first put in place. The assembly support cradle will have to remain in place until all of the modules have been placed on the saddle in order to insure stability.

- All of the forces between modules and acting on the saddles are less than the design forces that have been used to design the EB.

- Finally, a static analysis, identical to the one described above, was carried out for the addition of every module to the assembly from 14 modules in place to 64 modules in 
place. This analysis shows that with $14,15,16$ and 24, 25, 26, 27, 28 modules in place, the assembly is unstable, because an additional moment is needed at the bottom of the saddle in order to achieve stability. The support from the assembly cradle for modules $14,15,16$ is enough to provide stability, while a compression member between the back cryostat supports will be needed to provide stability for the top modules. 


\section{Appendix 1}

SADDLE FORCES AT THE BACK OF THE EB (TONS)

87 TON LOAD FROM THE BACK CRYOSTAT SUPPORT

IT IS ASSUMED THAT HALF OF THE MODULE WEIGHT

(5 TONS) IS SUPPORTED BY THE BACK SADDLE

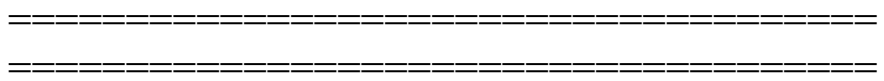

TOP SADDLE SUPPORT AT MODULE \# 7

BOTTOM SADDLE SUPPORT AT MODULE \# 6

\#MODULES $=7$

REACTION FORCES AT SADDLE

F1 $\quad$ F2 $\quad$ FS

$30.123 \quad 0.041 \quad 20.950$

LOCATION OF SADDLE REACTION POINT, LS $=2499.000$

TRUSS REACTION FORCE, F1 $=0.0000000 \mathrm{E}+00$

TOP SADDLE SUPPORT AT MODULE \# 8

BOTTOM SADDLE SUPPORT AT MODULE \# 6

\#MODULES $=8$

REACTION FORCES AT SADDLE

F1 F2 FS

$34.977 \quad 0.002 \quad 27.392$

LOCATION OF SADDLE REACTION POINT, LS $=2859.000$

TRUSS REACTION FORCE, F $1=0.0000000 \mathrm{E}+00$

TOP SADDLE SUPPORT AT MODULE \# 9

BOTTOM SADDLE SUPPORT AT MODULE \# 6

\#MODULES $=9$

REACTION FORCES AT SADDLE

F1 $\quad$ F2 $\quad$ FS

$31.680 \quad 8.278 \quad 32.335$

LOCATION OF SADDLE REACTION POINT, LS $=3000.000$

TRUSS REACTION FORCE, F1 $=0.0000000 \mathrm{E}+00$ 
TOP SADDLE SUPPORT AT MODULE \# 10

BOTTOM SADDLE SUPPORT AT MODULE \# 6

\#MODULES $=10$

REACTION FORCES AT SADDLE

F1 $\quad$ F2 $\quad$ FS

$26.701 \quad 18.214 \quad 35.928$

LOCATION OF SADDLE REACTION POINT, LS $=3000.000$

TRUSS REACTION FORCE, F1 $=0.0000000 \mathrm{E}+00$

TOP SADDLE SUPPORT AT MODULE \# 10

BOTTOM SADDLE SUPPORT AT MODULE \# 6

\#MODULES $=11$

REACTION FORCES AT SADDLE

F1 $\quad$ F2 $\quad$ FS

$29.371 \quad 20.036 \quad 39.521$

LOCATION OF SADDLE REACTION POINT, LS $=3000.000$

TRUSS REACTION FORCE, F $1=0.0000000 \mathrm{E}+00$

TOP SADDLE SUPPORT AT MODULE \# 10

BOTTOM SADDLE SUPPORT AT MODULE \# 6

\#MODULES $=12$

REACTION FORCES AT SADDLE

F1 $\quad$ F2 $\quad$ FS

$\begin{array}{lll}32.638 & 0.018 & 78.849\end{array}$

LOCATION OF SADDLE REACTION POINT, LS $=3000.000$

TRUSS REACTION FORCE, F1 = 41.39994

TOP SADDLE SUPPORT AT MODULE \# 10

BOTTOM SADDLE SUPPORT AT MODULE \# 6

\#MODULES=13

REACTION FORCES AT SADDLE

F1 $\quad$ F2 $\quad$ FS

$39.240 \quad 0.007 \quad 80.945$

LOCATION OF SADDLE REACTION POINT, LS $=3000.000$

TRUSS REACTION FORCE, F1 = 37.89999 
TOP SADDLE SUPPORT AT MODULE \# 10

BOTTOM SADDLE SUPPORT AT MODULE \# 6

\#MODULES $=14$

REACTION FORCES AT SADDLE

F1 $\quad$ F2 $\quad$ FS

$45.729 \quad 0.048 \quad 83.083$

LOCATION OF SADDLE REACTION POINT, LS $=3000.000$

TRUSS REACTION FORCE, F1 $=34.50005$

TOP SADDLE SUPPORT AT MODULE \# 10

BOTTOM SADDLE SUPPORT AT MODULE \# 6

\#MODULES $=15$

REACTION FORCES AT SADDLE

F1 $\quad$ F2 $\quad$ FS

$52.331 \quad 0.036 \quad 85.179$

LOCATION OF SADDLE REACTION POINT, LS $=3000.000$

TRUSS REACTION FORCE, $\mathrm{F} 1=31.00008$

TOP SADDLE SUPPORT AT MODULE \# 10

BOTTOM SADDLE SUPPORT AT MODULE \# 6

\#MODULES $=16$

REACTION FORCES AT SADDLE

$\begin{array}{lll}\text { F1 } & \text { F2 } & \text { FS }\end{array}$

$58.933 \quad 0.025 \quad 87.274$

LOCATION OF SADDLE REACTION POINT, LS $=3000.000$

TRUSS REACTION FORCE, F1 $=27.50007$

TOP SADDLE SUPPORT AT MODULE \# 10

BOTTOM SADDLE SUPPORT AT MODULE \# 6

\#MODULES $=17$

REACTION FORCES AT SADDLE

$\begin{array}{lll}\text { F1 } & \text { F2 } & \text { FS }\end{array}$

$\begin{array}{lll}65.535 & 0.013 \quad 89.370\end{array}$

LOCATION OF SADDLE REACTION POINT, LS $=3000.000$

TRUSS REACTION FORCE, F1 $=24.00006$ 
TOP SADDLE SUPPORT AT MODULE \# 10

BOTTOM SADDLE SUPPORT AT MODULE \# 6

\#MODULES $=18$

REACTION FORCES AT SADDLE

$\begin{array}{lll}\text { F1 } & \text { F2 } & \text { FS }\end{array}$

$72.137 \quad 0.002 \quad 91.465$

LOCATION OF SADDLE REACTION POINT, LS $=3000.000$

TRUSS REACTION FORCE, F1 $=20.50004$

TOP SADDLE SUPPORT AT MODULE \# 10

BOTTOM SADDLE SUPPORT AT MODULE \# 6

\#MODULES $=19$

REACTION FORCES AT SADDLE

$\begin{array}{lll}\text { F1 } & \text { F2 } & \text { FS }\end{array}$

$\begin{array}{lll}78.627 & 0.043 \quad 93.604\end{array}$

LOCATION OF SADDLE REACTION POINT, LS $=3000.000$

TRUSS REACTION FORCE, F1 = 17.10003

TOP SADDLE SUPPORT AT MODULE \# 10

BOTTOM SADDLE SUPPORT AT MODULE \# 6

\#MODULES $=20$

REACTION FORCES AT SADDLE

F1 $\quad$ F2 $\quad$ FS

$85.229 \quad 0.031 \quad 95.699$

LOCATION OF SADDLE REACTION POINT, LS $=3000.000$

TRUSS REACTION FORCE, $\mathrm{F} 1=13.60002$

TOP SADDLE SUPPORT AT MODULE \# 10

BOTTOM SADDLE SUPPORT AT MODULE \# 6

\#MODULES $=21$

REACTION FORCES AT SADDLE

F1 $\quad$ F2 $\quad$ FS

$91.831 \quad 0.020 \quad 97.795$

LOCATION OF SADDLE REACTION POINT, LS $=3000.000$

TRUSS REACTION FORCE, F1 $=10.10000$ 
TOP SADDLE SUPPORT AT MODULE \# 10

BOTTOM SADDLE SUPPORT AT MODULE \# 6

\#MODULES $=22$

REACTION FORCES AT SADDLE

F1 $\quad$ F2 $\quad$ FS

$\begin{array}{lll}98.433 & 0.009 & 99.890\end{array}$

LOCATION OF SADDLE REACTION POINT, LS $=3000.000$

TRUSS REACTION FORCE, F1 $=6.599996$

TOP SADDLE SUPPORT AT MODULE \# 10

BOTTOM SADDLE SUPPORT AT MODULE \# 6

\#MODULES $=23$

REACTION FORCES AT SADDLE

F1 $\quad$ F2 $\quad$ FS

$\begin{array}{lll}104.922 & 0.050 & 102.029\end{array}$

LOCATION OF SADDLE REACTION POINT, LS $=3000.000$

TRUSS REACTION FORCE, F1 = 3.199999

TOP SADDLE SUPPORT AT MODULE \# 10 BOTTOM SADDLE SUPPORT AT MODULE \# 6 \#MODULES $=24$

REACTION FORCES AT SADDLE

$\begin{array}{lll}\text { F1 } & \text { F2 } & \text { FS }\end{array}$

$111.187 \quad 0.195 \quad 104.253$

LOCATION OF SADDLE REACTION POINT, LS $=3000.000$

TRUSS REACTION FORCE, F1 $=0.0000000 \mathrm{E}+00$

TOP SADDLE SUPPORT AT MODULE \# 10

BOTTOM SADDLE SUPPORT AT MODULE \# 6

\#MODULES $=25$

REACTION FORCES AT SADDLE

$\begin{array}{lll}\text { F1 } & \text { F2 } & \text { FS }\end{array}$

$113.858 \quad 2.017 \quad 107.846$

LOCATION OF SADDLE REACTION POINT, $\mathrm{LS}=3000.000$

TRUSS REACTION FORCE, F1 $=0.0000000 \mathrm{E}+00$ 
TOP SADDLE SUPPORT AT MODULE \# 10 BOTTOM SADDLE SUPPORT AT MODULE \# 6 \#MODULES $=26$

REACTION FORCES AT SADDLE

F1 F2 FS

$\begin{array}{lll}116.528 & 3.838 & 111.438\end{array}$

LOCATION OF SADDLE REACTION POINT, LS $=3000.000$

TRUSS REACTION FORCE, F1 $=0.0000000 \mathrm{E}+00$

TOP SADDLE SUPPORT AT MODULE \# 10

BOTTOM SADDLE SUPPORT AT MODULE \# 6

\#MODULES $=27$

REACTION FORCES AT SADDLE

F1 $\quad$ F2 $\quad$ FS

$119.198 \quad 5.659 \quad 115.031$

LOCATION OF SADDLE REACTION POINT, LS $=3000.000$

TRUSS REACTION FORCE, F1 $=0.0000000 \mathrm{E}+00$

TOP SADDLE SUPPORT AT MODULE \# 10

BOTTOM SADDLE SUPPORT AT MODULE \# 6

\#MODULES $=28$

REACTION FORCES AT SADDLE

F1 $\quad$ F2 $\quad$ FS

$121.868 \quad 7.481 \quad 118.624$

LOCATION OF SADDLE REACTION POINT, LS $=3000.000$

TRUSS REACTION FORCE, F1 $=0.0000000 \mathrm{E}+00$

TOP SADDLE SUPPORT AT MODULE \# 10

BOTTOM SADDLE SUPPORT AT MODULE \# 6

\#MODULES=29

REACTION FORCES AT SADDLE

F1 $\quad$ F2 $\quad$ FS

$\begin{array}{lll}124.538 & 9.302 & 122.217\end{array}$

LOCATION OF SADDLE REACTION POINT, LS $=3000.000$

TRUSS REACTION FORCE, F1 $=0.0000000 \mathrm{E}+00$ 
TOP SADDLE SUPPORT AT MODULE \# 10

BOTTOM SADDLE SUPPORT AT MODULE \# 6

\#MODULES $=30$

REACTION FORCES AT SADDLE

F1 F2 FS

$127.208 \quad 11.124 \quad 125.810$

LOCATION OF SADDLE REACTION POINT, LS $=3000.000$

TRUSS REACTION FORCE, F1 $=0.0000000 \mathrm{E}+00$

TOP SADDLE SUPPORT AT MODULE \# 10

BOTTOM SADDLE SUPPORT AT MODULE \# 6

\#MODULES $=31$

REACTION FORCES AT SADDLE

$\begin{array}{lll}\text { F1 } & \text { F2 } & \text { FS }\end{array}$

$129.878 \quad 12.945 \quad 129.402$

LOCATION OF SADDLE REACTION POINT, LS $=3000.000$

TRUSS REACTION FORCE, F1 $=0.0000000 \mathrm{E}+00$

TOP SADDLE SUPPORT AT MODULE \# 10 BOTTOM SADDLE SUPPORT AT MODULE \# 6 \#MODULES $=32$

REACTION FORCES AT SADDLE

F1 $\quad$ F2 $\quad$ FS

$132.548 \quad 14.767 \quad 132.995$

LOCATION OF SADDLE REACTION POINT, LS $=3000.000$

TRUSS REACTION FORCE, F1 $=0.0000000 \mathrm{E}+00$ 


\section{Appendix 2 \\ Inner Radius Module Deflections Front of the EB}

$\begin{array}{cccc}\begin{array}{c}\text { Module } \\ \text { \# }\end{array} & \mathbf{X} & \mathbf{Y} & \mathbf{Z} \\ \mathbf{1} & 0.28 & -2.09 & -0.07 \\ \mathbf{2} & 0.27 & -2.02 & -0.09 \\ \mathbf{3} & 0.25 & -1.93 & -0.11 \\ \mathbf{4} & 0.20 & -1.80 & -0.12 \\ \mathbf{5} & 0.13 & -1.65 & -0.14 \\ \mathbf{6} & 0.03 & -1.46 & -0.16 \\ \mathbf{7} & 0.06 & -1.51 & -0.05 \\ \mathbf{8} & 0.09 & -1.55 & 0.07 \\ \mathbf{9} & 0.41 & -1.88 & 0.33 \\ \mathbf{1 0} & 0.94 & -2.33 & 0.64 \\ \mathbf{1 1} & 1.59 & -2.77 & 0.90 \\ \mathbf{1 2} & 2.22 & -3.14 & 1.12 \\ \mathbf{1 3} & 2.42 & -3.19 & 1.07 \\ \mathbf{1 4} & 2.57 & -3.24 & 1.04 \\ \mathbf{1 5} & 2.67 & -3.26 & 1.00 \\ \mathbf{1 6} & 2.71 & -3.27 & 0.95 \\ \mathbf{1 7} & 2.69 & -3.27 & 0.90 \\ \mathbf{1 8} & 2.62 & -3.28 & 0.83 \\ \mathbf{1 9} & 2.52 & -3.31 & 0.75 \\ \mathbf{2 0} & 2.38 & -3.35 & 0.67 \\ \mathbf{2 1} & 2.23 & -3.42 & 0.58 \\ \mathbf{2 2} & 2.06 & -3.51 & 0.49 \\ \mathbf{2 3} & 1.90 & -3.62 & 0.39 \\ \mathbf{2 4} & 1.75 & -3.75 & 0.28 \\ \mathbf{2 5} & 1.61 & -3.89 & 0.18 \\ \mathbf{2 6} & 1.49 & -4.03 & 0.07 \\ \mathbf{2 7} & 1.40 & -4.17 & -0.04 \\ \mathbf{2 8} & 1.33 & -4.31 & -0.15 \\ \mathbf{2 9} & 1.29 & -4.42 & -0.25 \\ \mathbf{3 0} & 1.26 & -4.51 & -0.36 \\ \mathbf{3 1} & 1.25 & -4.57 & -0.46\end{array}$




$\begin{array}{cccc}\begin{array}{c}\text { Module } \\ \mathbf{Z}\end{array} & \mathbf{X} & \mathbf{Y} & \mathbf{Z} \\ \mathbf{1} & 0.07 & -1.69 & 0.11 \\ \mathbf{2} & 0.07 & -1.63 & 0.11 \\ \mathbf{3} & 0.05 & -1.54 & 0.10 \\ \mathbf{4} & 0.01 & -1.42 & 0.10 \\ \mathbf{5} & -0.06 & -1.28 & 0.09 \\ \mathbf{6} & -0.15 & -1.10 & 0.08 \\ \mathbf{7} & -0.16 & -1.09 & 0.06 \\ \mathbf{8} & -0.17 & -1.08 & 0.04 \\ \mathbf{9} & -0.02 & -1.23 & 0.05 \\ \mathbf{1 0} & 0.24 & -1.44 & 0.07 \\ \mathbf{1 1} & 0.55 & -1.66 & 0.07 \\ \mathbf{1 2} & 0.84 & -1.81 & 0.07 \\ \mathbf{1 3} & 1.07 & -1.91 & 0.06 \\ \mathbf{1 4} & 1.24 & -1.96 & 0.05 \\ \mathbf{1 5} & 1.35 & -1.99 & 0.04 \\ \mathbf{1 6} & 1.40 & -2.00 & 0.03 \\ \mathbf{1 7} & 1.39 & -2.00 & 0.02 \\ \mathbf{1 8} & 1.34 & -2.01 & 0.01 \\ \mathbf{1 9} & 1.25 & -2.03 & 0.00 \\ \mathbf{2 0} & 1.12 & -2.07 & -0.01 \\ \mathbf{2 1} & 0.98 & -2.13 & -0.02 \\ \mathbf{2 2} & 0.83 & -2.21 & -0.03 \\ \mathbf{2 3} & 0.68 & -2.32 & -0.04 \\ \mathbf{2 4} & 0.54 & -2.43 & -0.05 \\ \mathbf{2 5} & 0.41 & -2.56 & -0.05 \\ \mathbf{2 6} & 0.30 & -2.70 & -0.06 \\ \mathbf{2 7} & 0.22 & -2.83 & -0.07 \\ \mathbf{2 8} & 0.15 & -2.95 & -0.08 \\ \mathbf{2 9} & 0.11 & -3.05 & -0.08 \\ \mathbf{3 0} & 0.09 & -3.12 & -0.09 \\ \mathbf{3 1} & 0.08 & -3.17 & -0.09\end{array}$




$\begin{array}{cccc}\begin{array}{c}\text { Module } \\ \text { \# }\end{array} & \mathbf{X} & \mathbf{Y} & \mathbf{Z} \\ \mathbf{1} & 0.15 & -1.81 & -0.08 \\ \mathbf{2} & 0.14 & -1.76 & -0.09 \\ \mathbf{3} & 0.13 & -1.68 & -0.11 \\ \mathbf{4} & 0.09 & -1.57 & -0.13 \\ \mathbf{5} & 0.03 & -1.43 & -0.14 \\ \mathbf{6} & -0.07 & -1.26 & -0.16 \\ \mathbf{7} & -0.06 & -1.28 & -0.07 \\ \mathbf{8} & -0.05 & -1.30 & 0.05 \\ \mathbf{9} & 0.21 & -1.57 & 0.31 \\ \mathbf{1 0} & 0.66 & -1.94 & 0.61 \\ \mathbf{1 1} & 1.21 & -2.32 & 0.87 \\ \mathbf{1 2} & 1.75 & -2.63 & 1.09 \\ \mathbf{1 3} & 1.87 & -2.64 & 1.04 \\ \mathbf{1 4} & 1.97 & -2.68 & 1.01 \\ \mathbf{1 5} & 2.04 & -2.69 & 0.97 \\ \mathbf{1 6} & 2.07 & -2.69 & 0.92 \\ \mathbf{1 7} & 2.06 & -2.69 & 0.86 \\ \mathbf{1 8} & 2.04 & -2.70 & 0.80 \\ \mathbf{1 9} & 2.00 & -2.70 & 0.72 \\ \mathbf{2 0} & 1.95 & -2.72 & 0.64 \\ \mathbf{2 1} & 1.91 & -2.74 & 0.56 \\ \mathbf{2 2} & 1.86 & -2.76 & 0.46 \\ \mathbf{2 3} & 1.83 & -2.78 & 0.37\end{array}$




$\begin{array}{cccc}\begin{array}{c}\text { Module } \\ \mathbf{\#}\end{array} & \mathbf{X} & \mathbf{Y} & \mathbf{Z} \\ \mathbf{1} & -0.05 & -1.39 & 0.09 \\ \mathbf{2} & -0.06 & -1.35 & 0.08 \\ \mathbf{3} & -0.07 & -1.27 & 0.08 \\ \mathbf{4} & -0.10 & -1.17 & 0.07 \\ \mathbf{5} & -0.16 & -1.04 & 0.07 \\ \mathbf{6} & -0.24 & -0.89 & 0.06 \\ \mathbf{7} & -0.27 & -0.85 & 0.05 \\ \mathbf{8} & -0.29 & -0.82 & 0.03 \\ \mathbf{9} & -0.20 & -0.91 & 0.04 \\ \mathbf{1 0} & -0.03 & -1.05 & 0.05 \\ \mathbf{1 1} & 0.19 & -1.20 & 0.06 \\ \mathbf{1 2} & 0.38 & -1.30 & 0.05 \\ \mathbf{1 3} & 0.54 & -1.37 & 0.04 \\ \mathbf{1 4} & 0.65 & -1.40 & 0.04 \\ \mathbf{1 5} & 0.73 & -1.42 & 0.03 \\ \mathbf{1 6} & 0.77 & -1.42 & 0.02 \\ \mathbf{1 7} & 0.78 & -1.42 & 0.02 \\ \mathbf{1 8} & 0.77 & -1.42 & 0.01 \\ \mathbf{1 9} & 0.75 & -1.43 & 0.00 \\ \mathbf{2 0} & 0.71 & -1.44 & -0.01 \\ \mathbf{2 1} & 0.68 & -1.45 & -0.01 \\ \mathbf{2 2} & 0.65 & -1.47 & -0.02 \\ \mathbf{2 3} & 0.63 & -1.48 & -0.03\end{array}$




$\begin{array}{cccc}\begin{array}{c}\text { Module } \\ \text { \# }\end{array} & \mathbf{X} & \mathbf{Y} & \mathbf{Z} \\ \mathbf{1} & 0.22 & -1.73 & -0.02 \\ \mathbf{2} & 0.21 & -1.68 & -0.04 \\ \mathbf{3} & 0.19 & -1.60 & -0.07 \\ \mathbf{4} & 0.15 & -1.49 & -0.09 \\ \mathbf{5} & 0.09 & -1.35 & -0.11 \\ \mathbf{6} & 0.00 & -1.19 & -0.14 \\ \mathbf{7} & -0.03 & -1.14 & -0.08 \\ \mathbf{8} & -0.08 & -1.09 & 0.00 \\ \mathbf{9} & 0.08 & -1.27 & 0.24 \\ \mathbf{1 0} & 0.41 & -1.54 & 0.53 \\ \mathbf{1 1} & 0.83 & -1.84 & 0.79 \\ \mathbf{1 2} & 1.25 & -2.09 & 1.00 \\ \mathbf{1 3} & 1.27 & -2.04 & 0.94 \\ \mathbf{1 4} & 1.28 & -2.05 & 0.91 \\ \mathbf{1 5} & 1.28 & -2.05 & 0.86 \\ \mathbf{1 6} & 1.27 & -2.05 & 0.81\end{array}$

$\begin{array}{cccc}\begin{array}{c}\text { Module } \\ \text { \# }\end{array} & \mathbf{X} & \mathbf{Y} & \mathbf{Z} \\ \mathbf{1} & 0.01 & -1.24 & 0.06 \\ \mathbf{2} & 0.01 & -1.20 & 0.06 \\ \mathbf{3} & -0.01 & -1.13 & 0.05 \\ \mathbf{4} & -0.04 & -1.02 & 0.05 \\ \mathbf{5} & -0.10 & -0.89 & 0.05 \\ \mathbf{6} & -0.18 & -0.74 & 0.04 \\ \mathbf{7} & -0.23 & -0.67 & 0.03 \\ \mathbf{8} & -0.29 & -0.59 & 0.02 \\ \mathbf{9} & -0.28 & -0.60 & 0.03 \\ \mathbf{1 0} & -0.22 & -0.65 & 0.03 \\ \mathbf{1 1} & -0.14 & -0.71 & 0.04 \\ \mathbf{1 2} & -0.07 & -0.75 & 0.03 \\ \mathbf{1 3} & -0.02 & -0.77 & 0.03 \\ \mathbf{1 4} & 0.01 & -0.77 & 0.03 \\ \mathbf{1 5} & 0.02 & -0.78 & 0.02 \\ \mathbf{1 6} & 0.02 & -0.78 & 0.02\end{array}$




$\begin{array}{cccc}\begin{array}{c}\text { Module } \\ \text { \# }\end{array} & \mathbf{X} & \mathbf{Y} & \mathbf{Z} \\ \mathbf{1} & -0.06 & -1.90 & 0.46 \\ \mathbf{2} & -0.07 & -1.83 & 0.40 \\ \mathbf{3} & -0.10 & -1.71 & 0.32 \\ \mathbf{4} & -0.16 & -1.54 & 0.20 \\ \mathbf{5} & -0.25 & -1.32 & 0.06 \\ \mathbf{6} & -0.39 & -1.06 & -0.11 \\ \mathbf{7} & -0.45 & -0.99 & -0.17 \\ \mathbf{8} & -0.52 & -0.92 & -0.22 \\ \mathbf{9} & -0.41 & -1.04 & -0.14 \\ \mathbf{1 0} & -0.17 & -1.25 & 0.01 \\ \mathbf{1 1} & 0.16 & -1.48 & 0.19 \\ \mathbf{1 2} & 0.52 & -1.70 & 0.39\end{array}$

$\begin{array}{cccc}\begin{array}{c}\text { Module } \\ \text { \# }\end{array} & \mathbf{X} & \mathbf{Y} & \mathbf{Z} \\ \mathbf{1} & 0.05 & -1.18 & 0.04 \\ \mathbf{2} & 0.39 & -0.49 & 0.04 \\ \mathbf{3} & 0.41 & -0.47 & 0.04 \\ \mathbf{4} & 0.41 & -0.48 & 0.03 \\ \mathbf{5} & 0.38 & -0.51 & 0.03 \\ \mathbf{6} & 0.30 & -0.60 & 0.04 \\ \mathbf{7} & 0.24 & -0.70 & 0.04 \\ \mathbf{8} & 0.16 & -0.84 & 0.04 \\ \mathbf{9} & 0.11 & -0.97 & 0.04 \\ \mathbf{1 0} & 0.07 & -1.07 & 0.05 \\ \mathbf{1 1} & 0.06 & -1.14 & 0.04 \\ \mathbf{1 2} & 0.05 & -1.17 & 0.04\end{array}$




\section{Appendix 3 \\ Inner Radius Module Deflections Back of the EB}

\begin{tabular}{cccc} 
Module \# & \multicolumn{2}{c}{ Coad Case EB1 } \\
$\mathbf{X}$ & $\mathbf{Y}$ & $\mathbf{Z}$ \\
$\mathbf{1}$ & 0.61 & -2.31 & -0.07 \\
$\mathbf{2}$ & 0.60 & -2.26 & -0.09 \\
$\mathbf{3}$ & 0.58 & -2.18 & -0.11 \\
$\mathbf{4}$ & 0.54 & -2.07 & -0.12 \\
$\mathbf{5}$ & 0.48 & -1.92 & -0.14 \\
$\mathbf{6}$ & 0.39 & -1.76 & -0.16 \\
$\mathbf{7}$ & 0.26 & -1.57 & -0.05 \\
$\mathbf{8}$ & 0.07 & -1.34 & 0.07 \\
$\mathbf{9}$ & -0.12 & -1.15 & 0.33 \\
$\mathbf{1 0}$ & -0.23 & -1.07 & 0.63 \\
$\mathbf{1 1}$ & -0.18 & -1.10 & 0.90 \\
$\mathbf{1 2}$ & -0.08 & -1.16 & 1.11 \\
$\mathbf{1 3}$ & 0.15 & -1.26 & 1.07 \\
$\mathbf{1 4}$ & 0.31 & -1.31 & 1.04 \\
$\mathbf{1 5}$ & 0.40 & -1.33 & 1.00 \\
$\mathbf{1 6}$ & 0.44 & -1.33 & 0.95 \\
$\mathbf{1 7}$ & 0.42 & -1.34 & 0.89 \\
$\mathbf{1 8}$ & 0.35 & -1.35 & 0.83 \\
$\mathbf{1 9}$ & 0.25 & -1.37 & 0.75 \\
$\mathbf{2 0}$ & 0.11 & -1.42 & 0.67 \\
$\mathbf{2 1}$ & -0.04 & -1.48 & 0.58 \\
$\mathbf{2 2}$ & -0.20 & -1.57 & 0.48 \\
$\mathbf{2 3}$ & -0.37 & -1.69 & 0.38 \\
$\mathbf{2 4}$ & -0.52 & -1.81 & 0.28 \\
$\mathbf{2 5}$ & -0.66 & -1.95 & 0.17 \\
$\mathbf{2 6}$ & -0.77 & -2.10 & 0.07 \\
$\mathbf{2 7}$ & -0.87 & -2.24 & -0.04 \\
$\mathbf{2 8}$ & -0.94 & -2.37 & -0.15 \\
$\mathbf{2 9}$ & -0.98 & -2.49 & -0.25 \\
$\mathbf{3 0}$ & -1.01 & -2.57 & -0.36 \\
$\mathbf{3 1}$ & -1.02 & -2.63 & -0.46
\end{tabular}




\section{Load Case EB2}

$\begin{array}{cccc}\text { Module \# } & \mathbf{X} & \mathbf{Y} & \mathbf{Z} \\ \mathbf{1} & 0.08 & -1.38 & 0.11 \\ \mathbf{2} & 0.07 & -1.34 & 0.11 \\ \mathbf{3} & 0.05 & -1.26 & 0.10 \\ \mathbf{4} & 0.02 & -1.15 & 0.10 \\ \mathbf{5} & -0.04 & -1.01 & 0.09 \\ \mathbf{6} & -0.13 & -0.85 & 0.08 \\ \mathbf{7} & -0.12 & -0.87 & 0.06 \\ \mathbf{8} & -0.11 & -0.89 & 0.04 \\ \mathbf{9} & 0.02 & -1.02 & 0.05 \\ \mathbf{1 0} & 0.23 & -1.19 & 0.07 \\ \mathbf{1 1} & 0.52 & -1.38 & 0.07 \\ \mathbf{1 2} & 0.80 & -1.54 & 0.06 \\ \mathbf{1 3} & 1.03 & -1.64 & 0.06 \\ \mathbf{1 4} & 1.20 & -1.69 & 0.05 \\ \mathbf{1 5} & 1.31 & -1.72 & 0.04 \\ \mathbf{1 6} & 1.36 & -1.72 & 0.03 \\ \mathbf{1 7} & 1.36 & -1.73 & 0.02 \\ \mathbf{1 8} & 1.30 & -1.74 & 0.01 \\ \mathbf{1 9} & 1.21 & -1.76 & 0.00 \\ \mathbf{2 0} & 1.09 & -1.80 & -0.01 \\ \mathbf{2 1} & 0.95 & -1.86 & -0.02 \\ \mathbf{2 2} & 0.80 & -1.94 & -0.03 \\ \mathbf{2 3} & 0.65 & -2.04 & -0.04 \\ \mathbf{2 4} & 0.50 & -2.16 & -0.05 \\ \mathbf{2 5} & 0.38 & -2.29 & -0.06 \\ \mathbf{2 6} & 0.27 & -2.43 & -0.06 \\ \mathbf{2 7} & 0.18 & -2.56 & -0.07 \\ \mathbf{2 8} & 0.12 & -2.67 & -0.08 \\ \mathbf{2 9} & 0.08 & -2.77 & -0.08 \\ \mathbf{3 0} & 0.06 & -2.85 & -0.09 \\ \mathbf{3 1} & 0.05 & -2.89 & -0.09\end{array}$




$\begin{array}{cccc}\text { Lodule \# } & \mathbf{X} & \mathbf{Y} & \mathbf{Z} \\ \mathbf{1} & 0.53 & -2.05 & -0.08 \\ \mathbf{2} & 0.52 & -2.01 & -0.10 \\ \mathbf{3} & 0.51 & -1.94 & -0.11 \\ \mathbf{4} & 0.48 & -1.84 & -0.13 \\ \mathbf{5} & 0.42 & -1.71 & -0.15 \\ \mathbf{6} & 0.34 & -1.57 & -0.16 \\ \mathbf{7} & 0.19 & -1.35 & -0.07 \\ \mathbf{8} & 0.00 & -1.11 & 0.05 \\ \mathbf{9} & -0.25 & -0.87 & 0.31 \\ \mathbf{1 0} & -0.43 & -0.72 & 0.60 \\ \mathbf{1 1} & -0.48 & -0.68 & 0.87 \\ \mathbf{1 2} & -0.47 & -0.69 & 1.07 \\ \mathbf{1 3} & -0.31 & -0.76 & 1.04 \\ \mathbf{1 4} & -0.21 & -0.79 & 1.01 \\ \mathbf{1 5} & -0.15 & -0.80 & 0.97 \\ \mathbf{1 6} & -0.12 & -0.80 & 0.92 \\ \mathbf{1 7} & -0.12 & -0.81 & 0.86 \\ \mathbf{1 8} & -0.15 & -0.81 & 0.80 \\ \mathbf{1 9} & -0.18 & -0.82 & 0.72 \\ \mathbf{2 0} & -0.23 & -0.83 & 0.64 \\ \mathbf{2 1} & -0.28 & -0.85 & 0.56 \\ \mathbf{2 2} & -0.32 & -0.87 & 0.46 \\ \mathbf{2 3} & -0.35 & -0.89 & 0.37\end{array}$




$\begin{array}{cccc}\text { Module \# } & \mathbf{X} & \mathbf{Y} & \mathbf{Z} \\ \mathbf{1} & -0.05 & -1.16 & 0.09 \\ \mathbf{2} & -0.05 & -1.13 & 0.08 \\ \mathbf{3} & -0.07 & -1.06 & 0.08 \\ \mathbf{4} & -0.10 & -0.96 & 0.07 \\ \mathbf{5} & -0.15 & -0.84 & 0.07 \\ \mathbf{6} & -0.23 & -0.69 & 0.06 \\ \mathbf{7} & -0.23 & -0.69 & 0.05 \\ \mathbf{8} & -0.24 & -0.67 & 0.03 \\ \mathbf{9} & -0.17 & -0.75 & 0.04 \\ \mathbf{1 0} & -0.03 & -0.86 & 0.05 \\ \mathbf{1 1} & 0.16 & -0.99 & 0.06 \\ \mathbf{1 2} & 0.35 & -1.09 & 0.05 \\ \mathbf{1 3} & 0.51 & -1.16 & 0.04 \\ \mathbf{1 4} & 0.62 & -1.19 & 0.04 \\ \mathbf{1 5} & 0.70 & -1.21 & 0.03 \\ \mathbf{1 6} & 0.74 & -1.21 & 0.02 \\ \mathbf{1 7} & 0.76 & -1.21 & 0.02 \\ \mathbf{1 8} & 0.75 & -1.21 & 0.01 \\ \mathbf{1 9} & 0.72 & -1.22 & 0.00 \\ \mathbf{2 0} & 0.69 & -1.23 & -0.01 \\ \mathbf{2 1} & 0.65 & -1.24 & -0.01 \\ \mathbf{2 2} & 0.62 & -1.26 & -0.02 \\ \mathbf{2 3} & 0.60 & -1.27 & -0.03\end{array}$




$\begin{array}{cccc}\text { Lodule \# } & \mathbf{X} & \mathbf{Y} & \mathbf{Z} \\ \mathbf{1} & 0.80 & -1.83 & -0.02 \\ \mathbf{2} & 0.79 & -1.79 & -0.05 \\ \mathbf{3} & 0.77 & -1.72 & -0.07 \\ \mathbf{4} & 0.74 & -1.61 & -0.09 \\ \mathbf{5} & 0.68 & -1.48 & -0.11 \\ \mathbf{6} & 0.60 & -1.33 & -0.14 \\ \mathbf{7} & 0.45 & -1.12 & -0.08 \\ \mathbf{8} & 0.26 & -0.88 & -0.01 \\ \mathbf{9} & -0.06 & -0.56 & 0.23 \\ \mathbf{1 0} & -0.37 & -0.31 & 0.53 \\ \mathbf{1 1} & -0.55 & -0.19 & 0.78 \\ \mathbf{1 2} & -0.66 & -0.13 & 0.98 \\ \mathbf{1 3} & -0.62 & -0.15 & 0.94 \\ \mathbf{1 4} & -0.61 & -0.15 & 0.91 \\ \mathbf{1 5} & -0.61 & -0.15 & 0.86 \\ \mathbf{1 6} & -0.62 & -0.15 & 0.81\end{array}$

\section{Load Case EB6}

$\begin{array}{cccc}\text { Module \# } & \mathbf{X} & \mathbf{Y} & \mathbf{Z} \\ \mathbf{1} & 0.02 & -1.09 & 0.06 \\ \mathbf{2} & 0.01 & -1.05 & 0.05 \\ \mathbf{3} & 0.00 & -0.99 & 0.05 \\ \mathbf{4} & -0.04 & -0.89 & 0.05 \\ \mathbf{5} & -0.09 & -0.76 & 0.04 \\ \mathbf{6} & -0.17 & -0.62 & 0.04 \\ \mathbf{7} & -0.21 & -0.56 & 0.03 \\ \mathbf{8} & -0.26 & -0.50 & 0.02 \\ \mathbf{9} & -0.26 & -0.50 & 0.03 \\ \mathbf{1 0} & -0.22 & -0.52 & 0.03 \\ \mathbf{1 1} & -0.15 & -0.57 & 0.04 \\ \mathbf{1 2} & -0.08 & -0.61 & 0.03 \\ \mathbf{1 3} & -0.04 & -0.63 & 0.03 \\ \mathbf{1 4} & -0.01 & -0.64 & 0.03 \\ \mathbf{1 5} & 0.00 & -0.64 & 0.02 \\ \mathbf{1 6} & 0.00 & -0.64 & 0.02\end{array}$




\begin{tabular}{cccc}
\multicolumn{4}{c}{ Load Case EB7 } \\
Module \# & $\mathbf{X}$ & $\mathbf{Y}$ & $\mathbf{Z}$ \\
$\mathbf{1}$ & 0.74 & -0.73 & 0.45 \\
$\mathbf{2}$ & 0.74 & -0.74 & 0.40 \\
$\mathbf{3}$ & 0.74 & -0.76 & 0.31 \\
$\mathbf{4}$ & 0.75 & -0.78 & 0.20 \\
$\mathbf{5}$ & 0.76 & -0.82 & 0.06 \\
$\mathbf{6}$ & 0.79 & -0.87 & -0.11 \\
$\mathbf{7}$ & 0.77 & -0.84 & -0.17 \\
$\mathbf{8}$ & 0.73 & -0.79 & -0.23 \\
$\mathbf{9}$ & 0.62 & -0.66 & -0.14 \\
$\mathbf{1 0}$ & 0.50 & -0.52 & 0.01 \\
$\mathbf{1 1}$ & 0.37 & -0.39 & 0.19 \\
$\mathbf{1 2}$ & 0.21 & -0.27 & 0.38
\end{tabular}

Load Case EB8

$\begin{array}{cccc}\text { Module \# } & \mathbf{X} & \mathbf{Y} & \mathbf{Z} \\ \mathbf{1} & 0.06 & -1.07 & 0.04 \\ \mathbf{2} & 0.41 & -0.49 & 0.04 \\ \mathbf{3} & 0.41 & -0.49 & 0.04 \\ \mathbf{4} & 0.38 & -0.52 & 0.03 \\ \mathbf{5} & 0.30 & -0.62 & 0.04 \\ \mathbf{6} & 0.24 & -0.71 & 0.05 \\ \mathbf{7} & 0.16 & -0.86 & 0.05 \\ \mathbf{8} & 0.11 & -0.98 & 0.05 \\ \mathbf{9} & 0.07 & -1.08 & 0.05 \\ \mathbf{1 0} & 0.06 & -1.15 & 0.05 \\ \mathbf{1 1} & 0.05 & -1.19 & 0.04 \\ \mathbf{1 2} & 0.05 & -1.15 & 0.04\end{array}$




\section{Appendix 4 \\ Outer Radius Module Deflections Front of the EB}

$\begin{array}{cccc}\text { Module \# } & \mathbf{X} & \mathbf{Y} & \mathbf{Y} \\ \mathbf{1} & 0.28 & -2.04 & 0.03 \\ \mathbf{2} & 0.34 & -1.94 & 0.03 \\ \mathbf{3} & 0.38 & -1.80 & 0.03 \\ \mathbf{4} & 0.39 & -1.62 & 0.02 \\ \mathbf{5} & 0.38 & -1.38 & 0.01 \\ \mathbf{6} & 0.39 & -1.22 & 0.00 \\ \mathbf{7} & 0.41 & -1.23 & -0.01 \\ \mathbf{8} & 0.49 & -1.42 & -0.03 \\ \mathbf{9} & 0.84 & -1.81 & -0.06 \\ \mathbf{1 0} & 1.39 & -2.28 & -0.09 \\ \mathbf{1 1} & 2.01 & -2.69 & -0.11 \\ \mathbf{1 2} & 2.41 & -2.92 & -0.10 \\ \mathbf{1 3} & 2.56 & -3.00 & -0.07 \\ \mathbf{1 4} & 2.67 & -3.05 & -0.04 \\ \mathbf{1 5} & 2.71 & -3.07 & -0.01 \\ \mathbf{1 6} & 2.70 & -3.07 & 0.02 \\ \mathbf{1 7} & 2.64 & -3.08 & 0.05 \\ \mathbf{1 8} & 2.54 & -3.10 & 0.07 \\ \mathbf{1 9} & 2.40 & -3.13 & 0.10 \\ \mathbf{2 0} & 2.24 & -3.19 & 0.13 \\ \mathbf{2 1} & 2.06 & -3.27 & 0.15 \\ \mathbf{2 2} & 1.88 & -3.38 & 0.18 \\ \mathbf{2 3} & 1.70 & -3.51 & 0.20 \\ \mathbf{2 4} & 1.54 & -3.65 & 0.22 \\ \mathbf{2 5} & 1.40 & -3.81 & 0.23 \\ \mathbf{2 6} & 1.28 & -3.97 & 0.25 \\ \mathbf{2 7} & 1.18 & -4.12 & 0.26 \\ \mathbf{2 8} & 1.11 & -4.26 & 0.27 \\ \mathbf{2 9} & 1.07 & -4.38 & 0.28 \\ \mathbf{3 0} & 1.05 & -4.48 & 0.28 \\ \mathbf{3 1} & 1.04 & -4.54 & 0.28\end{array}$




\begin{tabular}{|c|c|c|c|}
\hline \multicolumn{4}{|c|}{ Load Case EB2 } \\
\hline Module \# & $x$ & $\mathbf{Y}$ & $\mathbf{Z}$ \\
\hline 1 & 0.05 & -1.65 & -0.02 \\
\hline 2 & 0.08 & -1.57 & -0.01 \\
\hline 3 & 0.10 & -1.45 & -0.01 \\
\hline 4 & 0.09 & -1.30 & -0.01 \\
\hline 5 & 0.05 & -1.10 & -0.02 \\
\hline 6 & 0.02 & -0.94 & -0.02 \\
\hline 7 & 0.01 & -0.92 & -0.01 \\
\hline 8 & 0.02 & -1.00 & -0.01 \\
\hline 9 & 0.18 & -1.21 & -0.01 \\
\hline 10 & 0.45 & -1.44 & -0.01 \\
\hline 11 & 0.73 & -1.65 & 0.00 \\
\hline 12 & 0.97 & -1.78 & 0.00 \\
\hline 13 & 1.16 & -1.87 & 0.01 \\
\hline 14 & 1.30 & -1.91 & 0.01 \\
\hline 15 & 1.37 & -1.93 & 0.01 \\
\hline 16 & 1.39 & -1.94 & 0.02 \\
\hline 17 & 1.36 & -1.94 & 0.02 \\
\hline 18 & 1.28 & -1.95 & 0.02 \\
\hline 19 & 1.17 & -1.98 & 0.03 \\
\hline 20 & 1.03 & -2.03 & 0.03 \\
\hline 21 & 0.88 & -2.10 & 0.03 \\
\hline 22 & 0.72 & -2.19 & 0.03 \\
\hline 23 & 0.56 & -2.30 & 0.03 \\
\hline 24 & 0.42 & -2.43 & 0.03 \\
\hline 25 & 0.29 & -2.56 & 0.03 \\
\hline 26 & 0.19 & -2.70 & 0.03 \\
\hline 27 & 0.11 & -2.83 & 0.03 \\
\hline 28 & 0.05 & -2.95 & 0.03 \\
\hline 29 & 0.01 & -3.05 & 0.02 \\
\hline 30 & -0.01 & -3.12 & 0.02 \\
\hline 31 & -0.01 & -3.16 & 0.02 \\
\hline
\end{tabular}




\begin{tabular}{cccc} 
Module \# & \multicolumn{3}{c}{ Load Case EB3 } \\
$\mathbf{1}$ & $\mathbf{X}$ & $\mathbf{Y}$ & $\mathbf{Z}$ \\
$\mathbf{2}$ & 0.20 & -1.77 & 0.04 \\
$\mathbf{3}$ & 0.26 & -1.69 & 0.04 \\
$\mathbf{4}$ & 0.26 & -1.57 & 0.03 \\
$\mathbf{5}$ & 0.24 & -1.41 & 0.03 \\
$\mathbf{6}$ & 0.23 & -1.20 & 0.02 \\
$\mathbf{7}$ & 0.22 & -1.06 & 0.01 \\
$\mathbf{8}$ & 0.27 & -1.05 & 0.00 \\
$\mathbf{9}$ & 0.55 & -1.19 & -0.02 \\
$\mathbf{1 0}$ & 1.01 & -1.53 & -0.05 \\
$\mathbf{1 1}$ & 1.53 & -1.93 & -0.08 \\
$\mathbf{1 2}$ & 1.86 & -2.29 & -0.10 \\
$\mathbf{1 3}$ & 1.95 & -2.49 & -0.10 \\
$\mathbf{1 4}$ & 2.02 & -2.56 & -0.07 \\
$\mathbf{1 5}$ & 2.06 & -2.60 & -0.04 \\
$\mathbf{1 6}$ & 2.07 & -2.63 & -0.01 \\
$\mathbf{1 7}$ & 2.05 & -2.64 & 0.02 \\
$\mathbf{1 8}$ & 2.02 & -2.66 & 0.05 \\
$\mathbf{1 9}$ & 1.97 & -2.67 & 0.07 \\
$\mathbf{2 0}$ & 1.93 & -2.69 & 0.10 \\
$\mathbf{2 1}$ & 1.88 & -2.72 & 0.12 \\
$\mathbf{2 2}$ & 1.85 & -2.74 & 0.15 \\
$\mathbf{2 3}$ & 1.83 & -2.77 & 0.17 \\
$\mathbf{2 4}$ & 1.82 & -2.79 & 0.19 \\
& & -2.79 & 0.21
\end{tabular}




$\begin{array}{cccc}\text { Module \# } & \mathbf{X} & \mathbf{Y} & \\ \mathbf{1} & -0.03 & -1.37 & \mathbf{Z} \\ \mathbf{2} & -0.01 & -1.30 & -0.01 \\ \mathbf{3} & -0.02 & -1.20 & -0.01 \\ \mathbf{4} & -0.04 & -1.06 & -0.01 \\ \mathbf{5} & -0.08 & -0.90 & -0.01 \\ \mathbf{6} & -0.13 & -0.76 & -0.01 \\ \mathbf{7} & -0.16 & -0.73 & -0.01 \\ \mathbf{8} & -0.18 & -0.78 & -0.01 \\ \mathbf{9} & -0.09 & -0.92 & 0.00 \\ \mathbf{1 0} & 0.08 & -1.09 & 0.00 \\ \mathbf{1 1} & 0.28 & -1.24 & 0.00 \\ \mathbf{1 2} & 0.44 & -1.35 & 0.00 \\ \mathbf{1 3} & 0.57 & -1.42 & 0.00 \\ \mathbf{1 4} & 0.67 & -1.46 & 0.01 \\ \mathbf{1 5} & 0.74 & -1.49 & 0.01 \\ \mathbf{1 6} & 0.77 & -1.50 & 0.01 \\ \mathbf{1 7} & 0.78 & -1.51 & 0.01 \\ \mathbf{1 8} & 0.77 & -1.52 & 0.02 \\ \mathbf{1 9} & 0.76 & -1.54 & 0.02 \\ \mathbf{2 0} & 0.73 & -1.55 & 0.02 \\ \mathbf{2 1} & 0.71 & -1.56 & 0.02 \\ \mathbf{2 2} & 0.70 & -1.58 & 0.02 \\ \mathbf{2 3} & 0.70 & -1.58 & 0.02 \\ \mathbf{2 4} & 0.71 & -1.57 & 0.02 \\ & & & 0.02\end{array}$




\begin{tabular}{cccc}
\multicolumn{4}{c}{ Load Case EB5 } \\
Module \# & $\mathbf{X}$ & $\mathbf{Y}$ & $\mathbf{Z}$ \\
$\mathbf{1}$ & 0.26 & -1.69 & 0.05 \\
$\mathbf{2}$ & 0.30 & -1.61 & 0.05 \\
$\mathbf{3}$ & 0.33 & -1.49 & 0.05 \\
$\mathbf{4}$ & 0.33 & -1.33 & 0.04 \\
$\mathbf{5}$ & 0.30 & -1.13 & 0.04 \\
$\mathbf{6}$ & 0.27 & -0.96 & 0.04 \\
$\mathbf{7}$ & 0.22 & -0.88 & 0.03 \\
$\mathbf{8}$ & 0.21 & -0.93 & 0.01 \\
$\mathbf{9}$ & 0.40 & -1.15 & -0.02 \\
$\mathbf{1 0}$ & 0.75 & -1.45 & -0.05 \\
$\mathbf{1 1}$ & 1.14 & -1.72 & -0.07 \\
$\mathbf{1 2}$ & 1.35 & -1.84 & -0.07 \\
$\mathbf{1 3}$ & 1.34 & -1.86 & -0.04 \\
$\mathbf{1 4}$ & 1.33 & -1.86 & -0.02 \\
$\mathbf{1 5}$ & 1.30 & -1.87 & 0.01 \\
$\mathbf{1 6}$ & 1.27 & -1.87 & 0.03
\end{tabular}

\section{Load Case EB6}

$\begin{array}{cccc}\text { Module \# } & \mathbf{X} & \mathbf{Y} & \mathbf{Z} \\ \mathbf{1} & 0.04 & -1.22 & -0.01 \\ \mathbf{2} & 0.05 & -1.15 & -0.01 \\ \mathbf{3} & 0.05 & -1.05 & -0.01 \\ \mathbf{4} & 0.02 & -0.92 & -0.01 \\ \mathbf{5} & -0.03 & -0.76 & -0.01 \\ \mathbf{6} & -0.08 & -0.61 & -0.01 \\ \mathbf{7} & -0.14 & -0.53 & 0.00 \\ \mathbf{8} & -0.20 & -0.50 & 0.00 \\ \mathbf{9} & -0.19 & -0.54 & 0.00 \\ \mathbf{1 0} & -0.13 & -0.61 & 0.00 \\ \mathbf{1 1} & -0.07 & -0.67 & 0.00 \\ \mathbf{1 2} & -0.02 & -0.70 & 0.00 \\ \mathbf{1 3} & 0.01 & -0.72 & 0.00 \\ \mathbf{1 4} & 0.02 & -0.73 & 0.01 \\ \mathbf{1 5} & 0.02 & -0.74 & 0.01 \\ \mathbf{1 6} & 0.02 & -0.74 & 0.01\end{array}$




\begin{tabular}{cccc}
\multicolumn{4}{c}{ Load Case EB7 } \\
Module \# & $\mathbf{X}$ & $\mathbf{Y}$ & $\mathbf{Z}$ \\
$\mathbf{1}$ & -0.01 & -1.86 & 0.01 \\
$\mathbf{2}$ & 0.01 & -1.75 & 0.02 \\
$\mathbf{3}$ & 0.01 & -1.58 & 0.04 \\
$\mathbf{4}$ & -0.02 & -1.36 & 0.06 \\
$\mathbf{5}$ & -0.10 & -1.08 & 0.08 \\
$\mathbf{6}$ & -0.18 & -0.84 & 0.10 \\
$\mathbf{7}$ & -0.23 & -0.75 & 0.10 \\
$\mathbf{8}$ & -0.26 & -0.76 & 0.10 \\
$\mathbf{9}$ & -0.13 & -0.91 & 0.09 \\
$\mathbf{1 0}$ & 0.13 & -1.13 & 0.07 \\
$\mathbf{1 1}$ & 0.45 & -1.34 & 0.04 \\
$\mathbf{1 2}$ & 0.60 & -1.42 & 0.03
\end{tabular}

\section{Load Case EB8}

$\begin{array}{cccc}\text { Module \# } & \mathbf{X} & \mathbf{Y} & \mathbf{Z} \\ \mathbf{1} & 0.08 & -1.15 & -0.01 \\ \mathbf{2} & 0.34 & -0.38 & -0.01 \\ \mathbf{3} & 0.35 & -0.38 & -0.01 \\ \mathbf{4} & 0.32 & -0.39 & -0.01 \\ \mathbf{5} & 0.25 & -0.45 & 0.00 \\ \mathbf{6} & 0.17 & -0.55 & 0.00 \\ \mathbf{7} & 0.10 & -0.69 & 0.00 \\ \mathbf{8} & 0.05 & -0.85 & -0.01 \\ \mathbf{9} & 0.02 & -0.98 & -0.01 \\ \mathbf{1 0} & 0.02 & -1.08 & -0.01 \\ \mathbf{1 1} & 0.04 & -1.14 & -0.01 \\ \mathbf{1 2} & 0.06 & -1.16 & -0.01\end{array}$




\section{Appendix 5 \\ Outer Radius Module Deflections Back of the EB}

$\begin{array}{cccc}\begin{array}{c}\text { Module } \\ \mathbf{\#}\end{array} & \mathbf{X} & \mathbf{Y} & \mathbf{Z} \\ \mathbf{1} & 0.61 & -2.27 & 0.04 \\ \mathbf{2} & 0.65 & -2.20 & 0.04 \\ \mathbf{3} & 0.67 & -2.09 & 0.04 \\ \mathbf{4} & 0.67 & -1.93 & 0.04 \\ \mathbf{5} & 0.65 & -1.73 & 0.05 \\ \mathbf{6} & 0.58 & -1.51 & 0.05 \\ \mathbf{7} & 0.42 & -1.28 & 0.03 \\ \mathbf{8} & 0.17 & -1.06 & 0.00 \\ \mathbf{9} & -0.05 & -0.91 & -0.03 \\ \mathbf{1 0} & -0.11 & -0.90 & -0.07 \\ \mathbf{1 1} & -0.04 & -0.97 & -0.08 \\ \mathbf{1 2} & 0.10 & -1.05 & -0.09 \\ \mathbf{1 3} & 0.28 & -1.12 & -0.06 \\ \mathbf{1 4} & 0.39 & -1.16 & -0.03 \\ \mathbf{1 5} & 0.44 & -1.17 & -0.01 \\ \mathbf{1 6} & 0.43 & -1.17 & 0.02 \\ \mathbf{1 7} & 0.38 & -1.17 & 0.05 \\ \mathbf{1 8} & 0.28 & -1.18 & 0.07 \\ \mathbf{1 9} & 0.14 & -1.22 & 0.10 \\ \mathbf{2 0} & -0.02 & -1.27 & 0.12 \\ \mathbf{2 1} & -0.20 & -1.35 & 0.15 \\ \mathbf{2 2} & -0.38 & -1.45 & 0.17 \\ \mathbf{2 3} & -0.56 & -1.58 & 0.19 \\ \mathbf{2 4} & -0.72 & -1.72 & 0.21 \\ \mathbf{2 5} & -0.87 & -1.88 & 0.22 \\ \mathbf{2 6} & -0.99 & -2.04 & 0.24 \\ \mathbf{2 7} & -1.08 & -2.19 & 0.25 \\ \mathbf{2 8} & -1.15 & -2.33 & 0.26 \\ \mathbf{2 9} & -1.19 & -2.45 & 0.27 \\ \mathbf{3 0} & -1.22 & -2.55 & 0.28 \\ \mathbf{3 1} & -1.23 & -2.61 & 0.28\end{array}$




$\begin{array}{cccc}\begin{array}{c}\text { Module } \\ \text { Load Case EB2 }\end{array} & \\ \mathbf{1} & \mathbf{X} & \mathbf{Y} & \mathbf{Z} \\ \mathbf{2} & 0.06 & -1.35 & -0.01 \\ \mathbf{3} & 0.09 & -1.28 & -0.01 \\ \mathbf{4} & 0.11 & -1.18 & 0.00 \\ \mathbf{5} & 0.10 & -1.03 & 0.00 \\ \mathbf{6} & 0.07 & -0.84 & 0.01 \\ \mathbf{7} & 0.04 & -0.69 & 0.02 \\ \mathbf{8} & 0.07 & -0.71 & 0.02 \\ \mathbf{9} & 0.08 & -0.80 & 0.02 \\ \mathbf{1 0} & 0.20 & -0.97 & 0.02 \\ \mathbf{1 1} & 0.42 & -1.18 & 0.02 \\ \mathbf{1 2} & 0.69 & -1.37 & 0.02 \\ \mathbf{1 3} & 0.94 & -1.51 & 0.02 \\ \mathbf{1 4} & 1.13 & -1.60 & 0.02 \\ \mathbf{1 5} & 1.26 & -1.64 & 0.02 \\ \mathbf{1 6} & 1.34 & -1.66 & 0.02 \\ \mathbf{1 7} & 1.36 & -1.66 & 0.02 \\ \mathbf{1 8} & 1.32 & -1.67 & 0.02 \\ \mathbf{1 9} & 1.25 & -1.68 & 0.02 \\ \mathbf{2 0} & 1.00 & -1.75 & 0.02 \\ \mathbf{2 1} & 0.84 & -1.82 & 0.02 \\ \mathbf{2 2} & 0.68 & -1.92 & 0.02 \\ \mathbf{2 3} & 0.53 & -2.03 & 0.02 \\ \mathbf{2 4} & 0.38 & -2.15 & 0.02 \\ \mathbf{2 5} & 0.26 & -2.29 & 0.02 \\ \mathbf{2 6} & 0.15 & -2.43 & 0.02 \\ \mathbf{2 7} & 0.07 & -2.56 & 0.02 \\ \mathbf{2 8} & 0.01 & -2.68 & 0.02 \\ \mathbf{2 9} & -0.02 & -2.77 & 0.02 \\ \mathbf{3 0} & -0.04 & -2.85 & 0.02 \\ \mathbf{3 1} & -0.05 & -2.88 & 0.02\end{array}$




$\begin{array}{cccc}\begin{array}{c}\text { Module } \\ \mathbf{\#}\end{array} & \mathbf{X} & \mathbf{Y} & \mathbf{Z} \\ \mathbf{1} & 0.57 & -2.02 & 0.04 \\ \mathbf{2} & 0.59 & -1.96 & 0.04 \\ \mathbf{3} & 0.60 & -1.86 & 0.04 \\ \mathbf{4} & 0.59 & -1.72 & 0.05 \\ \mathbf{5} & 0.55 & -1.55 & 0.05 \\ \mathbf{6} & 0.47 & -1.35 & 0.04 \\ \mathbf{7} & 0.29 & -1.11 & 0.02 \\ \mathbf{8} & 0.01 & -0.87 & 0.00 \\ \mathbf{9} & -0.26 & -0.67 & -0.03 \\ \mathbf{1 0} & -0.41 & -0.60 & -0.07 \\ \mathbf{1 1} & -0.43 & -0.61 & -0.08 \\ \mathbf{1 2} & -0.36 & -0.66 & -0.08 \\ \mathbf{1 3} & -0.25 & -0.72 & -0.06 \\ \mathbf{1 4} & -0.17 & -0.76 & -0.03 \\ \mathbf{1 5} & -0.13 & -0.77 & -0.01 \\ \mathbf{1 6} & -0.12 & -0.78 & 0.02 \\ \mathbf{1 7} & -0.13 & -0.79 & 0.05 \\ \mathbf{1 8} & -0.17 & -0.80 & 0.07 \\ \mathbf{1 9} & -0.21 & -0.82 & 0.10 \\ \mathbf{2 0} & -0.25 & -0.84 & 0.12 \\ \mathbf{2 1} & -0.30 & -0.87 & 0.14 \\ \mathbf{2 2} & -0.33 & -0.89 & 0.17 \\ \mathbf{2 3} & -0.36 & -0.91 & 0.19 \\ \mathbf{2 4} & -0.36 & -0.91 & 0.21\end{array}$




$\begin{array}{cccc}\begin{array}{c}\text { Module } \\ \mathbf{\#}\end{array} & \mathbf{X} & \mathbf{Y} & \mathbf{Z} \\ \mathbf{1} & -0.02 & -1.14 & -0.01 \\ \mathbf{2} & -0.01 & -1.08 & -0.01 \\ \mathbf{3} & -0.01 & -0.99 & 0.00 \\ \mathbf{4} & -0.03 & -0.86 & 0.00 \\ \mathbf{5} & -0.07 & -0.70 & 0.01 \\ \mathbf{6} & -0.11 & -0.57 & 0.01 \\ \mathbf{7} & -0.12 & -0.57 & 0.02 \\ \mathbf{8} & -0.14 & -0.62 & 0.02 \\ \mathbf{9} & -0.08 & -0.74 & 0.02 \\ \mathbf{1 0} & 0.07 & -0.89 & 0.01 \\ \mathbf{1 1} & 0.25 & -1.04 & 0.01 \\ \mathbf{1 2} & 0.42 & -1.14 & 0.01 \\ \mathbf{1 3} & 0.55 & -1.21 & 0.01 \\ \mathbf{1 4} & 0.64 & -1.26 & 0.01 \\ \mathbf{1 5} & 0.71 & -1.28 & 0.01 \\ \mathbf{1 6} & 0.74 & -1.30 & 0.01 \\ \mathbf{1 7} & 0.76 & -1.31 & 0.02 \\ \mathbf{1 8} & 0.75 & -1.32 & 0.02 \\ \mathbf{1 9} & 0.73 & -1.33 & 0.02 \\ \mathbf{2 0} & 0.71 & -1.34 & 0.02 \\ \mathbf{2 1} & 0.69 & -1.36 & 0.02 \\ \mathbf{2 2} & 0.67 & -1.37 & 0.02 \\ \mathbf{2 3} & 0.67 & -1.37 & 0.02 \\ \mathbf{2 4} & 0.68 & -1.36 & 0.02\end{array}$




$\begin{array}{cccc}\begin{array}{c}\text { Module } \\ \mathbf{\#}\end{array} & \mathbf{X} & \mathbf{Y} & \mathbf{Z} \\ \mathbf{1} & 0.83 & -1.80 & 0.05 \\ \mathbf{2} & 0.86 & -1.74 & 0.05 \\ \mathbf{3} & 0.86 & -1.63 & 0.06 \\ \mathbf{4} & 0.85 & -1.49 & 0.06 \\ \mathbf{5} & 0.80 & -1.33 & 0.06 \\ \mathbf{6} & 0.71 & -1.12 & 0.06 \\ \mathbf{7} & 0.54 & -0.88 & 0.04 \\ \mathbf{8} & 0.25 & -0.59 & 0.03 \\ \mathbf{9} & -0.11 & -0.29 & -0.01 \\ \mathbf{1 0} & -0.37 & -0.11 & -0.04 \\ \mathbf{1 1} & -0.52 & -0.03 & -0.06 \\ \mathbf{1 2} & -0.58 & -0.01 & -0.06 \\ \mathbf{1 3} & -0.57 & -0.02 & -0.04 \\ \mathbf{1 4} & -0.57 & -0.02 & -0.01 \\ \mathbf{1 5} & -0.59 & -0.02 & 0.01 \\ \mathbf{1 6} & -0.62 & -0.02 & 0.04\end{array}$

$\begin{array}{cccc}\begin{array}{c}\text { Module } \\ \mathbf{\#}\end{array} & \mathbf{X} & \mathbf{Y} & \mathbf{Z} \\ \mathbf{1} & 0.04 & -1.07 & -0.01 \\ \mathbf{2} & 0.05 & -1.01 & 0.00 \\ \mathbf{3} & 0.05 & -0.91 & 0.00 \\ \mathbf{4} & 0.03 & -0.79 & 0.00 \\ \mathbf{5} & -0.02 & -0.63 & 0.00 \\ \mathbf{6} & -0.07 & -0.48 & 0.01 \\ \mathbf{7} & -0.11 & -0.43 & 0.01 \\ \mathbf{8} & -0.17 & -0.40 & 0.01 \\ \mathbf{9} & -0.18 & -0.42 & 0.01 \\ \mathbf{1 0} & -0.15 & -0.47 & 0.01 \\ \mathbf{1 1} & -0.09 & -0.53 & 0.01 \\ \mathbf{1 2} & -0.04 & -0.56 & 0.01 \\ \mathbf{1 3} & -0.01 & -0.59 & 0.01 \\ \mathbf{1 4} & 0.00 & -0.60 & 0.01 \\ \mathbf{1 5} & 0.00 & -0.60 & 0.01 \\ \mathbf{1 6} & 0.00 & -0.60 & 0.01\end{array}$




$\begin{array}{cccc}\begin{array}{c}\text { Load Case EB7 } \\ \text { Module }\end{array} & \mathbf{X} & \mathbf{Z} \\ \mathbf{\#} & \mathbf{Y} & \mathbf{Y} & 0.01 \\ \mathbf{1} & 0.78 & -0.73 & 0.03 \\ \mathbf{2} & 0.82 & -0.73 & 0.03 \\ \mathbf{3} & 0.86 & -0.73 & 0.05 \\ \mathbf{4} & 0.90 & -0.73 & 0.07 \\ \mathbf{5} & 0.96 & -0.74 & 0.10 \\ \mathbf{6} & 0.99 & -0.71 & 0.12 \\ \mathbf{7} & 0.97 & -0.66 & 0.12 \\ \mathbf{8} & 0.86 & -0.56 & 0.12 \\ \mathbf{9} & 0.71 & -0.44 & 0.10 \\ \mathbf{1 0} & 0.54 & -0.33 & 0.08 \\ \mathbf{1 1} & 0.37 & -0.23 & 0.05 \\ \mathbf{1 2} & 0.26 & -0.18 & 0.04\end{array}$

\section{Load Case EB8}

$\begin{array}{cccc}\begin{array}{c}\text { Module } \\ \text { \# }\end{array} & \mathbf{X} & \mathbf{Y} & \mathbf{Z} \\ \mathbf{1} & 0.08 & -1.04 & 0.00 \\ \mathbf{2} & 0.34 & -0.39 & -0.01 \\ \mathbf{3} & 0.34 & -0.38 & -0.01 \\ \mathbf{4} & 0.32 & -0.39 & -0.01 \\ \mathbf{5} & 0.26 & -0.44 & -0.01 \\ \mathbf{6} & 0.17 & -0.55 & -0.01 \\ \mathbf{7} & 0.10 & -0.68 & -0.01 \\ \mathbf{8} & 0.05 & -0.84 & -0.01 \\ \mathbf{9} & 0.03 & -0.98 & -0.01 \\ \mathbf{1 0} & 0.02 & -1.08 & -0.01 \\ \mathbf{1 1} & 0.04 & -1.15 & -0.01 \\ \mathbf{1 2} & 0.06 & -1.17 & -0.01\end{array}$

